\title{
Development and application of a machine learning algorithm for classification of elasmobranch behaviour from accelerometry data
}

\author{
L. R. Brewster ${ }^{1,2,3}$. J. J. Dale ${ }^{4}$ T. L. Guttridge ${ }^{1} \cdot$ S. H. Gruber ${ }^{1,5} \cdot$ A. C. Hansell ${ }^{6} \cdot$ M. Elliott $^{2} \cdot$ I. G. Cowx ${ }^{3}$. \\ N. M. Whitney ${ }^{7}$ - A. C. Gleiss ${ }^{8}$
}

Received: 29 August 2017 / Accepted: 31 January 2018 / Published online: 8 March 2018

(c) The Author(s) 2018. This article is an open access publication

\begin{abstract}
Discerning behaviours of free-ranging animals allows for quantification of their activity budget, providing important insight into ecology. Over recent years, accelerometers have been used to unveil the cryptic lives of animals. The increased ability of accelerometers to store large quantities of high resolution data has prompted a need for automated behavioural classification. We assessed the performance of several machine learning (ML) classifiers to discern five behaviours performed by accelerometer-equipped juvenile lemon sharks (Negaprion brevirostris) at Bimini, Bahamas $\left(25^{\circ} 44^{\prime} \mathrm{N}, 79^{\circ} 16^{\prime} \mathrm{W}\right)$. The sharks were observed to exhibit chafing, burst swimming, headshaking, resting and swimming in a semi-captive environment and these observations were used to ground-truth data for ML training and testing. ML methods included logistic regression, an artificial neural network, two random forest models, a gradient boosting model and a voting ensemble (VE) model, which combined the predictions of all other (base) models to improve classifier performance. The macro-averaged $F$-measure, an indicator of classifier performance, showed that the VE model improved overall classification ( $F$-measure 0.88$)$ above the strongest base learner model, gradient boosting (0.86). To test whether the VE model provided biologically meaningful results when applied to accelerometer data obtained from wild sharks, we investigated headshaking behaviour, as a proxy for prey capture, in relation to the variables: time of day, tidal phase and season. All variables were significant in predicting prey capture, with predations most likely to occur during early evening and less frequently during the dry season and high tides. These findings support previous hypotheses from sporadic visual observations.
\end{abstract}

Responsible Editor: J. Carlson.

Reviewed by Undisclosed experts.

Electronic supplementary material The online version of this article (https://doi.org/10.1007/s00227-018-3318-y) contains supplementary material, which is available to authorized users.

\section{R. Brewster}

lauran.brewster@gmail.com

1 Bimini Biological Field Station Foundation, South Bimini, Bahamas

2 Institute of Estuarine and Coastal Studies, University of Hull, Hull HU6 7RX, UK

3 Hull International Fisheries Institute, University of Hull, Hull HU6 7RX, UK

4 Department of Biology, Hopkins Marine Station, Stanford University, Pacific Grove, CA 93950, USA

5 Division of Marine Biology and Fisheries, Rosenstiel School of Marine and Atmospheric Science, 4600 Rickenbacker Causeway, Miami, FL 33149, USA

\section{Introduction}

Selecting the optimal behavioural response can increase individual fitness, have adaptive significance and evolutionary consequences (Lima and Dill 1990; McNamara and Houston 1996; Shepard et al. 2008b). Identification

6 Department of Fisheries Oceanography, School for Marine Science and Technology, University of Massachusetts Dartmouth, 836 South Rodney French Blvd, New Bedford, MA 02719, USA

7 Anderson Cabot Center for Ocean Life, New England Aquarium, Central Wharf, Boston, MA 02110, USA

8 Centre For Fish and Fisheries Research, School of Veterinary and Life Sciences, Murdoch University, 90 South Street, Perth, WA 6150, Australia 
of these behaviours as well as their subsequent energetic costs can provide insight into an individual's activity budget and ecology (Cooke et al. 2004; Metcalfe et al. 2016), in turn impacting populations (Forsman 2015). Identifying and understanding natural behaviours of free-ranging animals is particularly challenging for species living in aquatic environments, because continuous direct observations are impossible to gather (Gleiss et al. 2011a; Brown et al. 2013). If marine animals are to be observed in their natural environment, new techniques need to be developed to monitor them over long periods, in poor visibility (e.g., low light levels or turbid water), at deeper depths and in adverse environmental conditions. Biotelemetry (transmitted biological data) and biologging tools (archival tags; see Cooke 2008 for further details) capable of overcoming such obstacles are now widely available (Cooke et al. 2004; Rutz and Hays 2009; Bograd et al. 2010). One such tool is the acceleration data logger (ADL), a device that measures changes in velocity and can be used to determine body orientation and kinematics for behavioural classification (Shepard et al. 2008b; Sakamoto et al. 2009; Gleiss et al. 2011b; Brown et al. 2013). Data are stored in the device's on-board memory. These ADLs must be retrieved to obtain data, but allow data to be recorded at higher frequencies, providing insight into fine-scale behaviour. Their application has become increasingly popular for use on animals occupying media that preclude direct observations, and now many ADLs are coupled with additional sensors for monitoring abiotic factors (e.g., temperature and depth, Watanabe et al. 2012; Wright et al. 2014; Lear et al. 2017; Carroll et al. 2014).

Modern ADLs collect large quantities of high resolution acceleration and abiotic data, making deciphering behaviours from acceleration data manually, as was done initially, impractical. This has prompted a need for more automated behaviour classification (Shepard et al. 2008a; Tanha et al. 2012; Bidder et al. 2014), through machinelearning algorithms and the development of new software (Sakamoto et al. 2009; Walker et al. 2015). Machine learning (ML) can be broadly categorised into supervised and unsupervised (Hastie et al. 2009; Valletta et al. 2017), both of which have strengths and weaknesses. In supervised learning, a training set is required whereby the input (e.g., acceleration features) and associated outcome measure/label (e.g., behaviour) are known. Once the input variables can be appropriately mapped to the outcome, the algorithm can be used to make predictions from new input data (Hastie et al. 2009). Examples of these techniques include decision trees, random forest (RF), K-nearest neighbour and linear discriminant analysis (Kiani et al. 1998; Staudenmayer et al. 2009; Nathan et al. 2012; Soltis et al. 2012; Campbell et al. 2013; Bidder et al. 2014; Resheff et al. 2014; Williams et al. 2015; Sur et al. 2017). Supervised ML has been applied to classify acceleration data in many studies and has the advantage of clearly defined behaviours and simple interpretation (LeosBarajas et al. 2017). However, it demands a comprehensive training data set which can be unattainable for some species and requires a validation process (Allen et al. 2016). Selection of the optimum supervised ML method can also be time-consuming (Ladds et al. 2017). Clustering algorithms such as $k$-means clustering and principal component analysis, where no outcome measure is provided, are examples of unsupervised learning methods (Sakamoto et al. 2009; Valletta et al. 2017). The algorithm groups data based on inherent similarities between input variables (Hastie et al. 2009). Unsupervised learning has the potential to reveal novel behavioural patterns (Battaile et al. 2015; Sakamoto et al. 2009; Chimienti et al. 2016) and is particularly valuable for species that are not readily adaptable in captivity or are not easily observed in the wild, hindering direct observation during data collection (i.e., ground-truthing). However, in the case of $k$-means clustering, drawbacks include a priori specification of the number of behaviours reflected in the dataset by setting the number of clusters. The optimum number of clusters can often be ambiguous with too few clusters resulting in similar behaviours being grouped together, whilst too many may artificially separate behaviours (Sakamoto et al. 2009; Whitney et al. 2010; Gleiss et al. 2017; Valletta et al. 2017). Some ML algorithms such as artificial neural networks (ANN) and hidden Markov models can be used in both a supervised and unsupervised learning context (Schmidhuber 2015; Leos-Barajas et al. 2017).

Ensemble classifiers combine the strengths of multiple supervised machine learners (base learners), to improve overall prediction accuracy of the model (ratio of correct predictions over number of total predictions, Hastie et al. 2009). Ladds et al. (2017) classified acceleration data obtained on captive fur seals and sea lions into behavioural categories using super learning — a form of ensemble learning — whereby the output of the base learners is used as additional data to inform the super-learning algorithm. Their optimum model achieved superior accuracy $(85.1 \%$ for four behavioural categories) and lower variance than any of the constituent models. Dutta et al. (2015) tested three ensemble classifier techniques: Adaboost, Random Subspace and bagging, finding the latter could achieve $96 \%$ accuracy in classifying acceleration data from cattle into five classes. Voting ensemble classifiers are one of the simplest to implement and the decision rule can be based on the majority vote, averaging probabilities or product of probabilities (see Catal et al. 2015 for comparison of results between methods). However, we are not aware of the application of this form of ensemble classifier to acceleration data obtained on non-humans or of ensemble classifiers for predicting behavioural states for animals at liberty.

Whilst ML techniques have been applied to classify acceleration data obtained from a variety of terrestrial fauna (e.g., vultures, Nathan et al. 2012; cheetahs, Grünewälder 
et al. 2012; badgers, McClune et al. 2014; pumas, Wang et al. 2015; cows, Martiskainen et al. 2009; Diosdado et al. 2015; condors, Williams et al. 2015) and some air-breathing marine fauna (e.g., cetaceans, Allen et al. 2016; Owen et al. 2016; pinnipeds, Battaile et al. 2015; Ladds et al. 2017; and penguins, Yoda et al. 1999; Carroll et al. 2014; Chessa et al. 2017), their use on elasmobranch acceleration data has been limited to two unsupervised methods (Whitney et al. 2010; Leos-Barajas et al. 2017). Sharks regularly occupy high trophic positions (Cortés 1999; Estrada et al. 2003) and can influence the structure of marine ecosystems (Heithaus et al. 2008; Rasher et al. 2017; Barley et al. 2017). However, their typically high mobility and inaccessible habitat make their natural behaviour difficult or impossible to observe directly (Klimley et al. 1992; Nakamura et al. 2011; Watanabe et al. 2012; Payne et al. 2016). To date, accelerometer application with sharks has provided new information on activity patterns (Whitney et al. 2007; Gleiss et al. 2013; Leos-Barajas et al. 2017; Gleiss et al. 2017), mating behaviour (Whitney et al. 2010), metabolic demands (Gleiss et al. 2010; Barnett et al. 2016; Whitney et al. 2016b; Bouyoucos et al. 2017; Lear et al. 2017), post-release mortality (Whitney et al. 2016a) and biomechanics (Gleiss et al. 2011a; Payne et al. 2016; Papastamatiou et al. 2018). However, in sharks, behavioural classification has relied upon visual inspection of the data or unsupervised ML methods (Whitney et al. 2010; Leos-Barajas et al. 2017), where overall classification performance cannot be quantified.

Despite the above applications of ADLs to study sharks, this technology has not yet been used to investigate their feeding behaviour. Many sharks are thought to be predominantly opportunistic, asynchronous feeders (Wetherbee et al. 1990; Newman et al. 2010). Knowledge of the feeding ecology of a species, including feeding frequency and periodicity, is required for developing ecosystem models and predicting the impact of population decline (Stevens et al. 2000). Through stomach eversions and digestion analysis, Bush (2003) showed that juvenile scalloped hammerhead sharks (Sphyrna lewini) are nocturnal hunters. Higher quantities of food were also found in their stomachs during the winter, but this could be the result of slower gastric evacuation at reduced temperatures rather than an increase in consumption. Accelerometers may provide a simpler, more accurate and less invasive method for investigating feeding frequency and periodicity than stomach content analysis and allow identification of behaviours for a single animal over extended time periods rather than single point measures.

Here a tool is developed to classify shark behaviour from accelerometry data, using the juvenile lemon shark (Negaprion brevirostris) as a model species due to its hardiness in captivity, its abundance, and high site fidelity to nursery grounds at the study site, Bimini, Bahamas (Gruber 1982; Morrissey and Gruber 1993). This study used ADLs, semi-captive behavioural observations and ML algorithms to accomplish three objectives: (1) obtain ground-truthed behavioural observations of accelerometer-equipped lemon sharks; (2) use ground-truthed data to generate and assess the performance of supervised ML algorithms to predict wild lemon shark behaviour, and (3) explore the applicability of these predictions in relation to abiotic factors to gain insight into the behavioural ecology of the juvenile lemon shark.

\section{Materials and methods}

\section{Tag package}

The tag package consisted of a triaxial acceleration data logger (G6a+ ADL; $40 \mathrm{~mm} \times 28 \mathrm{~mm} \times 17 \mathrm{~mm}, 30 \mathrm{~Hz}, 56 \mathrm{MB}$, CEFAS Technologies Ltd) coupled, using epoxy resin, with an acoustic transmitter (Sonotronics PT-4; $9 \mathrm{~mm} \times 25 \mathrm{~mm}$, 134-136 dB, battery life: 3 months, Sonotronics Inc) for tag retrieval via acoustic telemetry. It was attached to individuals by puncturing two holes ( $1.5 \mathrm{~mm}$ diameter), using a hypodermic needle, through the base of the first dorsal fin and passing nylon monofilament through the ADL and the fin (Sundström et al. 2001; Gleiss et al. 2009a; Lear et al. 2017). On the opposite side of the dorsal fin, the ends of the monofilament were secured against two small plastic plates using stainless steel crimps. A medical-grade polyurethane foam (Poron Blue Medical Grade 4708, $1.5 \mathrm{~mm}$ thick, unabraded) was placed between the plastic plates and the ADL to minimise rubbing and skin damage.

\section{Captive trials}

This study was conducted around the Bimini Islands, Bahamas $\left(25^{\circ} 44^{\prime} \mathrm{N}, 79^{\circ} 16^{\prime} \mathrm{W}\right)$, two small mangrove fringed islands approximately $85 \mathrm{~km}$ due east of Miami, Florida, USA. The system has been extensively studied by the Bimini Biological Field Station Foundation and provides well-documented nursery grounds for juvenile lemon sharks (Chapman et al. 2009). For captive trials, juvenile lemon sharks were caught $(n=4)$ using a $180 \mathrm{~m} \times 2 \mathrm{~m}$ monofilament gillnet set perpendicular to the shoreline of South Bimini (Gruber et al. 2001; Table 1). To reduce the risk of mortality, nets were checked at 15-min intervals or when a disturbance was detected. Previously captured individuals were identified using an intramuscular passive integrated transponder (PIT; Destron Fearing Inc.; Gruber et al. 2001) injected under the skin at the base of the dorsal fin. Sharks of appropriate size [75-90 cm total length (TL)] were transported to a nearby rectangular semi-enclosed pen $(10 \times 6 \mathrm{~m})$ erected on the neighbouring sand flats. The minimum TL was dictated by the size and mass of the tag package, whilst the maximum TL represented the largest animals that could be housed in a respirometer for a separate component of 
Table 1 Juvenile lemon sharks that exhibited the five behaviours for classification during semi-captive trials for development of an acceleration ethogram

\begin{tabular}{llll}
\hline PIT tag ID & Sex & $\begin{array}{l}\text { Total length } \\
(\mathrm{cm})\end{array}$ & Weight $(\mathrm{kg})$ \\
\hline 985121031792723 & Female & 82.6 & 3.75 \\
4C4A2D3A12 & Female & 80.5 & 3.10 \\
4C3A6C313A & Male & 79.2 & 3.15 \\
4C3B312275 & Male & 85.2 & 3.75 \\
\hline
\end{tabular}

a larger study (Lear et al. 2017). A large rectangular pen was used to minimise the repetitive circular swimming patterns previously observed when housing juvenile lemon sharks in a circular pen (Gleiss et al. 2009a). The pens were constructed from plastic diamond mesh, allowing the sharks to experience natural ambient conditions (i.e., salinity, temperature, tides, lunar cycles; Guttridge 2009). Except during trials, animals were fed to satiation with thawed or fresh fish every third day (Wetherbee et al. 1987; Cortés and Gruber 1994; Guttridge et al. 2009). To allow sharks the opportunity to recover and to acclimatize to the pen, ADL packages were attached after a period of at least 2 days within the pen. Behavioural trials to develop an ethogram (i.e., a catalogue of distinct activities constituting the behavioural repertoire of an animal; Grier 1984; Sakamoto et al. 2009) began 24-h after ADL attachment.

To view captive sharks, a wooden tower (height $3 \mathrm{~m}$ ) was placed next to the pen. Observers recorded behaviour/body movements to the second using digital clocks synchronized with the ADL. These observations were paired with the acceleration measurements to be used in developing a classification algorithm for predicting behaviour from individuals at liberty. Juvenile lemon sharks have been observed swimming, chafing (flashing), resting, burst-swimming and feeding around the Bimini Islands. Therefore, we focused on obtaining acceleration signatures for these behaviours during captive trials. Burst events performed in the wild were witnessed in response to disturbances (e.g., passing boats), predators, and during hunting. Successful prey capture in the wild was accompanied by sideto-side headshakes (authors' pers. obs). Chafing, a behaviour hypothesised as a method for dislodging parasites (Myrberg Jr and Gruber 1974), was characterised by a roll motion whereby the dorsal surface of the shark came into contact with the substrate or water surface. Resting behaviour was defined as individuals lying motionless on the seabed. To train the classifiers, more replicates of rare behaviours were needed than were readily displayed in the pens and subsequently some behaviours were induced. For example, burst events were prompted by throwing dive weights to the side or behind an individual or by making large movements next to the pen. Following completion of the ethogram trial, the shark was recaptured using a dip-net and the ADL package was removed. Individuals were monitored for several days prior to release.

\section{Data collection from free-ranging lemon sharks}

Juvenile lemon sharks are known to frequent a mangrove inlet at Bimini, Bahamas on a daily basis and being a shallow and sheltered area it provides opportunity to deploy ADLs and conduct observations of wild shark behaviours (Guttridge 2009; Guttridge et al. 2012). Individuals were captured using a dip-net as they exited the mangrove inlet on the ebbing tide. They were transported to a small research vessel anchored several metres away from the entrance to the inlet, and placed in a circular tub (1 m diameter) for tag attachment and collection of morphometric data (i.e., TL $(\mathrm{cm})$, weight $(\mathrm{kg})$ and sex; Table 2). All individuals were scanned for presence of a PIT tag and newly captured sharks had a PIT tag inserted. Following tag attachment, the shark was manually carried from the research vessel and released in the direction to which it was heading prior to capture. The duration of capture to release was $\sim 5 \mathrm{~min}$. ADLs were attached approximately $24 \mathrm{~h}$ prior to the commencement of data logging and recorded data (acceleration $30 \mathrm{~Hz}$; pressure and temperature $1 \mathrm{~Hz}$ ) for $120 \mathrm{~h}$. A 24-h delay of the commencement of logging by the tag allowed for post-release recovery, increasing the chances to record normal behaviour.

Various techniques were employed to recapture tagged individuals: the mangrove inlet was seined off over high tides as per the capture process; stationary baited gillnets were placed across the flats at various locations and checked every $15 \mathrm{~min}$ or when there was a disturbance, whilst boats with acoustic tracking gear scanned the area listening for PT-4 transmitters. Sharks located by acoustic telemetry would be encircled with a gillnet, and the tags were removed upon capture. The ADL attachment site healed in $<30$ days with recaptured animals displaying no apparent marks in subsequent seasons.

\section{Data analysis}

ADL data analysis was conducted in Igor Pro version 6.34 (WaveMetrics Inc, Lake Oswego, Oregon, USA). Static acceleration, which measures the orientation of the accelerometer in relation to the earth's gravitational pull, representing animal posture, was extracted from the acceleration data (as recorded by the ADL) using $3 \mathrm{~s}$ box smoothing (Shepard et al. 2008a). After separation, dynamic acceleration, a measure of the animals' movement, remained for each orthogonal axis: surge, heave and sway (x, y, z; Fig. 1). Surge denotes anterior-posterior movement, heave represents dorsal-ventral movement and sway is lateral movement. Typical routine swimming is characterised by regular oscillations in the dynamic swaying acceleration of sharks, representing individual tail-beats (Gleiss et al. 2009a, b). Overall dynamic body acceleration (ODBA) was calculated as the sum of the absolute dynamic axes values (Wilson 
Table 2 Wild lemon sharks tagged with the accelerometer data logger/acoustic transmitter package. Seasons are split into wet (April-September; $n=10$ ) and dry season (OctoberMarch; $n=10$ )

\begin{tabular}{|c|c|c|c|c|c|}
\hline Pit tag\# & Season & Sex & Dates & $\begin{array}{l}\text { Total length } \\
\text { (TL) }(\mathrm{cm})\end{array}$ & Weight (kg) \\
\hline $4 \mathrm{~A} 0 \mathrm{~A} 043 \mathrm{D} 40^{\mathrm{a}}$ & Wet & F & 29/07/12-03/08/12 & 77.5 & 3.25 \\
\hline 4A73536511 & Wet & F & $29 / 07 / 12-03 / 08 / 12$ & 83.6 & 3.00 \\
\hline 4A66401437 & Wet & $\mathrm{F}$ & $29 / 07 / 12-03 / 08 / 12$ & 78.1 & 2.80 \\
\hline 4A44545C6C & Wet & M & $31 / 08 / 12-05 / 09 / 12$ & 82.4 & 3.25 \\
\hline $4 \mathrm{~A} 63380105^{\mathrm{b}}$ & Wet & F & $31 / 08 / 12-05 / 09 / 12$ & 81.4 & 2.75 \\
\hline 4C3B211816 & Wet & F & $31 / 08 / 12-05 / 09 / 12$ & 81.0 & 2.10 \\
\hline 4B7B473332 & Wet & $\mathrm{F}$ & $31 / 08 / 12-05 / 09 / 12$ & 83.1 & 3.40 \\
\hline 4A68061232 & Wet & M & $31 / 08 / 12-05 / 09 / 12$ & 74.3 & 2.60 \\
\hline 4C3B086000 & Wet & M & $31 / 08 / 12-05 / 09 / 12$ & 76.0 & 2.10 \\
\hline 985121031823859 & Wet & $\mathrm{F}$ & $29 / 08 / 14-03 / 09 / 14$ & 77.5 & 2.30 \\
\hline 4B7B442028 & Dry & M & $12 / 01 / 13-17 / 01 / 13$ & 80.6 & 2.75 \\
\hline $4 \mathrm{~A} 63380105^{\mathrm{b}}$ & Dry & $\mathrm{F}$ & $12 / 01 / 13-17 / 01 / 13$ & 82.5 & 3.50 \\
\hline 4A603C232D & Dry & M & $12 / 01 / 13-17 / 01 / 13$ & 87.3 & 3.25 \\
\hline 4C3B2A712D & Dry & F & $26 / 03 / 14-31 / 03 / 14$ & 88.3 & 3.17 \\
\hline $4 \mathrm{~A} 0 \mathrm{~A} 043 \mathrm{D} 40^{\mathrm{a}}$ & Dry & $\mathrm{F}$ & $26 / 03 / 14-31 / 03 / 14$ & 89.0 & 4.10 \\
\hline 4С 3B032B0C & Dry & F & $26 / 03 / 14-31 / 03 / 14$ & 86.5 & 3.10 \\
\hline 4A5A577669 & Dry & M & $08 / 11 / 14-13 / 11 / 14$ & 88.4 & 3.60 \\
\hline 4B7B464873 & Dry & M & 08/11/14-13/11/14 & 87.1 & 3.50 \\
\hline 4C497D6463 & Dry & F & $08 / 11 / 14-13 / 11 / 14$ & 78.4 & 2.40 \\
\hline 4C4A736341 & Dry & $\mathrm{F}$ & 08/11/14-13/11/14 & 81.7 & 3.00 \\
\hline
\end{tabular}

${ }^{\mathrm{a}, \mathrm{b}}$ Indicate individuals tagged during both seasons
Fig. 1 a Examples of the five behaviours for classification. Overall dynamic body acceleration (ODBA) is calculated as the sum of the absolute values of dynamic acceleration from the three axes. b Dynamic acceleration in the three orthogonal axes: sway (blue), heave (red) and surge (grey) during each behaviour and $\mathbf{c}$ corresponding wavelet spectrum generated from the sway axis showing increased signal strength amplitude during the burst and headshake event

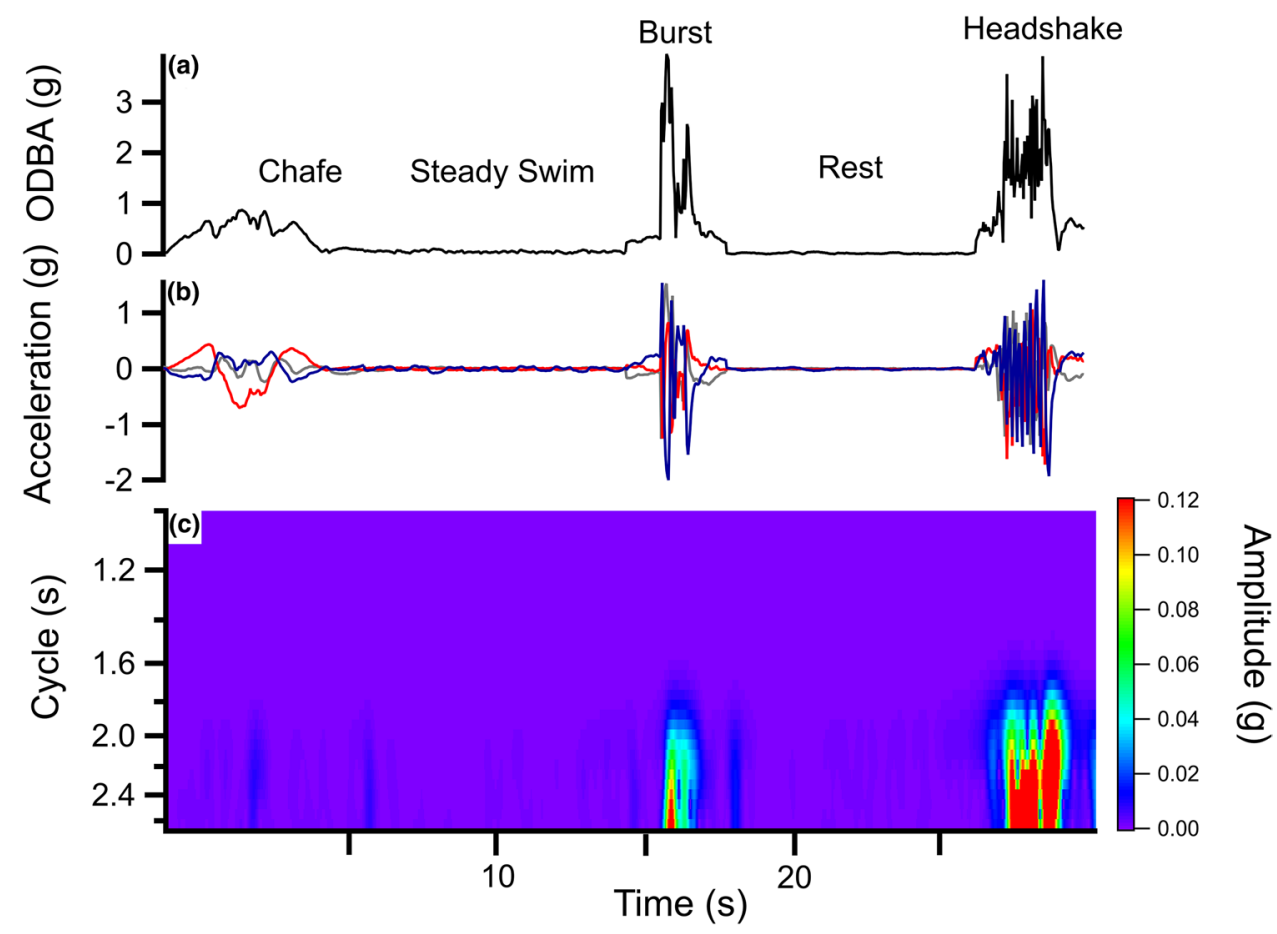

et al. 2006). The signal strength amplitude and frequency of the dominant cycle from the sway axis was extracted using continuous wavelet transformation, with the Morlet mother wavelet function through Ethographer v2.0 (Sakamoto et al.
2009; available from http://bre.soc.i.kyoto-u.ac.jp/bls/index .php?Ethographer; Fig. 1). These feature vectors form part of the acceleration summary statistics calculated for use as predictor variables in this multi-class classification scenario 
(Table 3; Nathan et al. 2012; Zheng et al. 2013; Wang et al. 2015).

\section{Classifier development}

To classify the behaviour of wild sharks into the five behavioural categories established during the captive ethogram trials, an ensemble classifier model was built using ML base models from the 'scikit-learn' package (Pedregosa et al. 2011) in Python (Python Software Foundation, Python Language Reference, version 2.7; available at http://www. python.org). The ground-truthed data were split into three portions: (1) the training set $(60 \%)$ for developing all base learner models; (2) the validation set $(20 \%)$ for model selection as well as weighting; and (3) the test set (20\%) to estimate the generalisation error and overall performance of the selected final model (Hastie et al. 2009). The data splits were randomized and implemented using stratified sampling in the 'scikit-learn' package to preserve the relative class frequencies in each data set. For each observation, base models generated the probability that the observation belonged to each class. As such, only classification models capable of generating probabilities (rather than only class labels) were considered for the ensemble classifier built here (henceforth referred to as voting ensemble; VE). The array of probabilities predicted for each observation and class were then averaged across all of the models selected during the validation stage, and the class with the highest predicted probability was selected as the final predicted class value.

The best performing base learners, as established from confusion matrices, were selected for the VE using the validation set. They include logistic regression (LR), a multilayer perceptron artificial neural network (ANN), two random forest (RF) models and a gradient tree boosting (GB) model. The following section provides a brief overview of the theory underlying each base learner model selected.

The LR model used a 'one-vs-all' technique. This reduces a multiclass scenario into multiple binary ones, where a logistic model is created for each class versus all remaining classes (Rifkin and Klautau 2004). For new data, each model provides a probability estimate of an observation, with the observation being assigned to the class with the highest probability score.

ANNs are a non-linear regression or classification technique used to model the relationship between predictors and a response variable (Staudenmayer et al. 2009). Multilayer perceptrons are feed-forward ANNs. Input data are mapped onto known output nodes/classes in the final layer, through hidden layers of nodes using a non-linear activation function. In this instance, one hidden layer was implemented with 100 nodes. Each node is connected to nodes in the subsequent layer, but with different connection weights

Table 3 Features extracted from acceleration data loggers and used to train the base learner classifiers (see Zheng et al. 2013 for equations)

\begin{tabular}{|c|c|c|}
\hline Parameter & Label & Definition \\
\hline Static acceleration & Xstat, Ystat, Zstat & $\begin{array}{l}\text { Static acceleration for each axis reflective of body } \\
\text { orientation }\end{array}$ \\
\hline Dynamic acceleration & Xdyn, Ydyn, Zdyn & $\begin{array}{l}1 \mathrm{~s} \text { means of body movement generated acceleration } \\
\text { in each axis }\end{array}$ \\
\hline Overall Dynamic Body Acceleration & ODBA & $\begin{array}{l}\text { Sum of the absolute values from the three dynamic } \\
\text { axis }\end{array}$ \\
\hline Amplitude & Amp & $\begin{array}{l}\text { Amplitude of the signal derived from the sway axis } \\
\text { body movement }\end{array}$ \\
\hline Frequency & $\mathrm{Hz}$ & $\begin{array}{l}\text { Dominant tailbeat frequency from lateral accelera- } \\
\text { tion }\end{array}$ \\
\hline Standard deviation & $\begin{array}{l}\text { XstatSD, YstatSD, ZstatSD, XdynSD, YdynSD, } \\
\text { ZdynSD, ODBASD }\end{array}$ & $\begin{array}{l}\text { Standard deviation of static and dynamic accelera- } \\
\text { tion measures in each axis }\end{array}$ \\
\hline Skewness & $\begin{array}{l}\text { XstatSkew, YstatSkew, ZstatSkew, XdynSkew, } \\
\text { YdynSkew, ZdynSkew, ODBASkew }\end{array}$ & A measure of the symmetry of the feature vector \\
\hline Kurtosis & $\begin{array}{l}\text { XstatKurt, YstatKurt, ZstatKurt, XdynKurt, } \\
\text { YdynKurt, ZdynKurt, ODBAKurt }\end{array}$ & A measure of the tail shape of the feature vector \\
\hline Maximum & $\begin{array}{l}\text { XstatMax, YstatMax, ZstatMax, XdynMax, Ydyn- } \\
\text { Max, ZdynMax, ODBAMax }\end{array}$ & $\begin{array}{l}\text { Maximum values per second for dynamic and static } \\
\text { acceleration in each axis and for ODBA }\end{array}$ \\
\hline Minimum & $\begin{array}{l}\text { XstatMin, YstatMin, ZstatMin, XdynMin, Ydyn- } \\
\text { Min, ZdynMin, ODBAMin }\end{array}$ & $\begin{array}{l}\text { Minimum values per second for dynamic and static } \\
\text { acceleration in each axis and for ODBA }\end{array}$ \\
\hline Frequencies from wavelet spectra & X.values & $\begin{array}{l}\text { Amplitude for the relevant frequency obtained } \\
\text { through the continuous wavelet transformation } \\
\text { generated spectrogram }\end{array}$ \\
\hline
\end{tabular}

Static acceleration was calculated from the raw acceleration using 3-s box smoothing, leaving dynamic acceleration remaining. Overall dynamic body acceleration (ODBA) is calculated as the sum of the absolute values of dynamic acceleration from the three axes 
reflecting the importance of the connections. At first, these weights are randomly assigned. The predicted output is compared to the known output and the error between them is passed backwards through the layers, adjusting the connection weights between nodes accordingly. This is known as backpropagation and is a process that is repeated until the model error is deemed to be at an acceptable level. The softmax function, a generalized logistic function, is applied as the output layer to allow for multi-class classification with probability estimates.

$\mathrm{RF}$ analysis is a leading ML algorithm that has been applied successfully to accelerometer data for behaviour recognition in a variety of species (Casale et al. 2011; Graf et al. 2015; Luštrek and Kaluža 2009; Nathan et al. 2012; Wang et al. 2015). RF is a 'supervised ensemble classifier' in itself, whereby many un-pruned classification trees are generated, with each tree voting for a class. RF incorporates two levels of randomness to minimise overfitting: (1) a bootstrap sample of data (62.3\%) are used to generate every tree and (2) at each tree node, a subset of predictor variables (m) is selected at random to encourage tree diversity. The remaining data, not used in the bootstrap sample, are used to determine the misclassification rate (Breiman et al. 1984). In most cases, the prediction is made by majority vote from all trees within 'the forest', however, the 'scikit-learn' implementation averages the probabilistic prediction from each classifier to generate a final prediction. For the VE, two RF models were generated from the training data, differing by the split criterion used for choosing the best splitting attribute at each node, i.e., the Gini impurity (model referred to forthwith as RFG), and entropy (RFE) which measures information gained and is most commonly used in classification scenarios.

GB is another ensemble learning method whereby a forward stage-wise additive model is built (Friedman 2001). Unlike RF where each tree is grown extensively, in GB, the trees are very shallow (e.g., they may only have one spilt and is then termed a 'decision stump'). Weak decision trees are iteratively built, optimising the parameters of the most recent tree, whilst maintaining the parameters of earlier trees to reduce over-fitting. Subsequent trees focus on earlier incorrect predictions, trying to correct those and minimise the deviance loss function (Hastie et al. 2009). In this study, 100 base learner decision trees were fitted with a maximum tree depth of three.

Whilst the predictive power of ANN, GB and RF ML techniques is often improved over simple decision trees, they are commonly referred to as 'black box' algorithms, since their decision-making rules are difficult to interpret (Hastie et al. 2009). However, GB and RF do allow for relative ranking of predictor variable importance (Breiman et al. 1984). This allows insight into the features most influencing classification and can be used for variable selection where there are many variables.

\section{Evaluation metrics}

Metrics calculated from the confusion matrix include precision, recall and the $F$-measure and are commonly used to judge the quality of a classification model (Chen et al. 2004; Özgür et al. 2005). They are calculated from the true positive (TP), false positive (FP) and false negative (FN) values in the confusion matrix. TPs are those that have been correctly assigned to their class, and therefore equal the number in the row and column cell corresponding to the class in question. FPs are those that are incorrectly classified to a class and therefore, in a multiclass classifier, are found by summing the values in the class column, excluding TP. FNs are those that belong to a class but have not been assigned to it and are calculated by summing the values of the class row, excluding the TP. From these values, several indices of performance can be calculated (Özgür et al. 2005) and used to determine the macro-averaged $F$-measure for evaluating overall classification performance. The performances indices are as follows:

Recall: the ratio of correctly identified classes to all known correct classes (Eq. 1):

$\mathrm{Re}=\frac{\mathrm{TP}_{i}}{\mathrm{TP}_{i}+\mathrm{FN}_{i}}$.

Precision: the fraction of correctly identified classes (i.e., correct recall) against all predicted classes (Eq. 2). A classification model may have good recall for a class if many known observations are correctly identified, but poor precision if this is accompanied by many observations being incorrectly assigned to that class (i.e., a high number of FNs; Sokolova and Lapalme 2009).

$\mathrm{Pr}=\frac{\mathrm{TP}_{i}}{\mathrm{TP}_{i}+\mathrm{FP}_{i}}$.

$F$-measure: the harmonic mean of recall and precision for each class (Eq. 3).

$F_{i}=\frac{2 \operatorname{Pr}_{i} \operatorname{Re}_{i}}{\operatorname{Pr}_{i}+\operatorname{Re}_{i}}$.

Macro-average $F$-measure: the mean of the $F$-measures determined for each class (Eq. 4).

$F($ macro-averaged $)=\frac{\sum_{i=1}^{M} F_{i}}{M}$

$M$, in Eq. 4 represents the number of classes in the classification problem. Both the $F$-measure and macro-averaged $F$-measure are represented by a value in the range $0-1$, with larger values representing improved classification quality. In this study, the optimal model was selected using the 
macro-averaged $F$-measure. This metric gives equal weight to all classes, regardless of case frequency (Özgür et al. 2005).

\section{Classifier application}

The VE model was used to predict the behavioural class for each second of ADL data obtained in the wild. A single successful predation event was variable in duration and sometimes incorporated intermittent headshaking. Therefore, the behaviour was considered either present or absent per hour of tag deployment to ensure each predation event contributed equally to the dataset. As all visually observed headshakes in captivity lasted for a minimum of $2 \mathrm{~s}$, headshakes that lasted less than two consecutive seconds were filtered from the data to minimise the impact of false positive readings (Carroll et al. 2014). All hours that included remaining headshakes were subsequently defined as headshaking being present.

Generalized additive mixed models (GAMMs) are a semiparametric approach used for modelling effects in response to a variety of predictor variables (Hastie and Tibshirani 1990). A GAMM with a binomial distribution was employed to model the absence or presence of headshaking behaviour of juvenile lemon sharks (Table 4).

Co-linearity of covariates was investigated using generalized variance-inflation factor (GVIF) scores. Any covariate with a score greater than three was removed and the GVIFs were recalculated (Zuur and Ieno 2012). High and low tidal phases were considered to be $1 \mathrm{~h}$ either side of peak high and low tide, with flood and ebb phases occupying the times in between. The tides in the Bimini lagoon and the refuge spot are known to lag approximately $1 \mathrm{~h}$ behind those at NOAA's North Bimini station (ID: TEC4617) and tidal phase was calculated accordingly (Guttridge et al. 2012). Shark ID was incorporated as a random effect to avoid pseudo-replication. The modelling was implemented using the 'gamm4' package in $\mathrm{R}$ (version 3.3.2). Significance was determined at the 0.05 level. The optimum model was selected using log-likelihood scores, which measure the lack of fit (Johnson and Omland 2004; Wasserman 2000). Scores closest to zero represent optimal fit.

\section{Results}

\section{Captive trials}

Resting occurred on six occasions $(73.3 \pm 107.4 \mathrm{~s}$; range 12-291 s) during captive trials. Chafing behaviour occurred naturally in the pens by individuals with $(4.6 \pm 1.6 \mathrm{~s}, n=58$; range $3-11 \mathrm{~s}$ ) and without tag packages. Thirty-five burst events were recorded $(1.3 \pm 0.5 \mathrm{~s}$; range $1-3 \mathrm{~s})$. Feeding occurred sporadically in the pen and was usually accompanied by side-to-side headshakes, a movement that was not witnessed outside of prey capture. Eight instances of feeding occurred by ADL equipped sharks in the pen, all on their preferred prey species (yellow fin mojarra; Gerres cinereus), and seven of which elicited headshaking. The single feeding event that did not result in headshaking consisted of a gulping motion on a smaller fish and was not discernible from swimming behaviour by the dorsally mounted ADL. As such, prey manipulation period was defined here as the duration between the commencement and cessation of headshaking for a single prey item. Successful prey manipulation events varied in duration from 2 to $559 \mathrm{~s}$. Headshaking occurred intermittently for a total of $113 \mathrm{~s}$ during prey manipulation. In $50 \%$ of instances, the shark did not consume the whole prey item after one headshake, but continued to hold the prey in its mouth or dropped it and displayed further headshaking upon re-collecting it (Fig. 2).

\section{Model development and performance}

The confusion matrices presented in Table 5 were used to calculate the evaluation metrics for all base learners and the VE model (Table 6). As the GB model performed best of all the base learner models during the validation stage, it was weighted three times more than other models in the VE. This marginally improved the macro-averaged $F$-measure by 0.005 . The remaining models were weighted once and had to agree confidently in their predictions to override a differing prediction from the GB model. Subsequently, the final VE output is similar to the GB model, with a few erroneous

Table 4 Covariates included in binomial generalized additive mixed model investigating headshake events in juvenile lemon sharks in Bimini, Bahamas

\begin{tabular}{llll}
\hline Variable & Range & Description & Variable Type \\
\hline Time of day & $0-23 \mathrm{~h}$ & 24-h day & Cyclic smoother \\
Season & Dry/wet & Season sharks were tagged & Categorical \\
Tidal phase & Ebb-Low-Flood-High & Tidal phase based on NOAA's tidal charts. High and low tide & Categorical \\
Shark ID & $1-20$ & were categorised as one hour either side of event & Random effect \\
\hline
\end{tabular}


Fig. 2 An example from the sway acceleration axis of a $63 \mathrm{~s}$ prey manipulation event, consisting of three headshakes (HS; totalling $19 \mathrm{~s}$ ) and a brief burst event

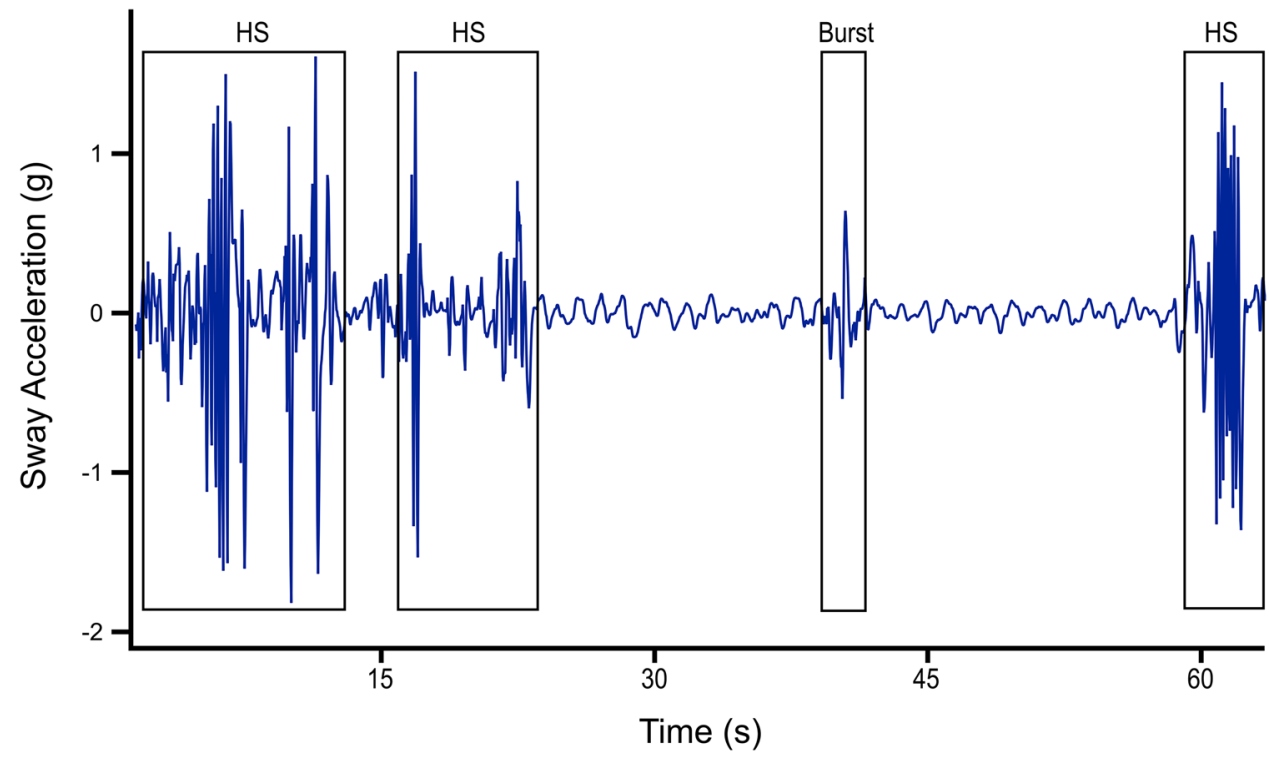

predictions corrected for improved performance. For the swim class, the ANN and GB models provided the optimum recall, with the RF classifiers providing the lowest recall values, but slightly higher precision. The RF and GB models all obtained a precision value of 1 for resting behaviour and obtained the highest recall values ranging from 0.955 to 0.966 . LR obtained the lowest value for both precision $(0.973)$ and recall $(0.830)$ in this class. The ANN model provided the poorest recall value for the chafe class but the highest precision (0.957). GB supplied the next highest precision value (0.927) along with the best recall (0.944), whilst the RFG model yielded the worst precision value (0.831).

Events from the burst and headshake classes had the highest-class errors (Table 5), yielding the lowest overall $F$-measures of all the classes from the VE (0.737 and 0.791, respectively). The RF models correctly classified the most instances for headshaking behaviour, but the improved recall was at the expense of precision, with these two models also obtaining the lowest precision rates for this class. The GB model obtained the next highest recall value, 0.696 , with precision improved two-fold over the best performing RF model. The highest recall value for burst behaviour was 0.800 , obtained by the ANN model; however, the precision value was also the second lowest of all models (0.533). The GB model contributed the best precision value, whilst the LR model performed poorly in both metrics for this class, yielding the lowest class $F$-measure overall (Table 6).

An increase in the class $F$-measure for chafe, burst and headshake classes indicated that they particularly benefitted from the VE technique. Resting was the only class to show a decreased $F$-measure from the VE when compared to the best performing base learners for that class (RF models), however, this difference was small (i.e., 0.977 vs 0.983 ). The macro-averaged $F$-measure indicated that the VE model improved overall classification above the strongest base learner model (VE: 0.888, GB: 0.856) and showed considerable improvement over the LR model, which performed the weakest of those included after the model validation stage (0.723).

All feature vectors were included in the base learner models. The relative importance of these features varied between the GB and RF models, although both models identified mean ODBA as an important metric (Fig. S1).

\section{Classifier application}

Behavioural classifications were applied to $2400 \mathrm{~h}$ of accelerometry data obtained in the wild $(n=18)$. Headshake predictions were then used to gain insight into temporal dynamics of foraging behaviour. GVIF scores $(>3)$ revealed collinearity between temperature and season. Temperature was removed as a covariate in favour of season, as all deployments occurred in two distinct seasons and observations suggested feeding increased in the warmer, wet season. The time series included in the GAMM did not show significant auto-correlation and subsequently did not require an auto-correlation structure. Covariates season, tidal phase and time of day were included in the optimal model (Table 7). All covariates were significant in predicting the presence of successful predation events for the juvenile lemon shark in Bimini (Table 8). Presence of hourly headshakes varied, with the dominant peak occurring around $1700 \mathrm{~h}$ and a smaller peak around $0230 \mathrm{~h}$ (Fig. 3). Headshakes occurred less frequently over high tide and during the dry season (Table 8). 
Table 5 Confusion matrix generated for the test set of the ground-truthed data

\begin{tabular}{|c|c|c|c|c|c|c|c|c|}
\hline & \multirow[t]{2}{*}{ Model } & \multicolumn{7}{|c|}{ Predicted behaviours } \\
\hline & & Class & Swim & HS & Rest & Chafe & Burst & Class error \\
\hline \multirow[t]{30}{*}{ Actual behaviours } & \multirow[t]{5}{*}{ LR } & Swim & 6984 & 6 & 2 & 8 & 0 & 0.002 \\
\hline & & HS & 4 & 16 & 0 & 1 & 2 & 0.304 \\
\hline & & Rest & 15 & 0 & 73 & 0 & 0 & 0.170 \\
\hline & & Chafe & 3 & 3 & 0 & 48 & 0 & 0.111 \\
\hline & & Burst & 0 & 8 & 0 & 0 & 2 & 0.800 \\
\hline & \multirow[t]{5}{*}{ ANN } & Swim & 6995 & 2 & 1 & 2 & 0 & 0.001 \\
\hline & & HS & 6 & 11 & 0 & 0 & 6 & 0.522 \\
\hline & & Rest & 7 & 0 & 81 & 0 & 0 & 0.080 \\
\hline & & Chafe & 6 & 3 & 0 & 44 & 1 & 0.185 \\
\hline & & Burst & 0 & 2 & 0 & 0 & 8 & 0.200 \\
\hline & \multirow[t]{5}{*}{ RFG } & Swim & 6969 & 20 & 0 & 9 & 2 & 0.004 \\
\hline & & HS & 1 & 19 & 0 & 1 & 2 & 0.174 \\
\hline & & Rest & 3 & 0 & 85 & 0 & 0 & 0.034 \\
\hline & & Chafe & 2 & 2 & 0 & 49 & 1 & 0.093 \\
\hline & & Burst & 0 & 3 & 0 & 0 & 7 & 0.300 \\
\hline & \multirow[t]{5}{*}{ RFE } & Swim & 6968 & 24 & 0 & 8 & 0 & 0.005 \\
\hline & & HS & 1 & 18 & 0 & 1 & 3 & 0.217 \\
\hline & & Rest & 3 & 0 & 85 & 0 & 0 & 0.034 \\
\hline & & Chafe & 2 & 3 & 0 & 48 & 1 & 0.111 \\
\hline & & Burst & 0 & 3 & 0 & 0 & 7 & 0.300 \\
\hline & \multirow[t]{5}{*}{ GB } & Swim & 6995 & 2 & 0 & 3 & 0 & 0.001 \\
\hline & & HS & 5 & 16 & 0 & 1 & 1 & 0.304 \\
\hline & & Rest & 4 & 0 & 84 & 0 & 0 & 0.045 \\
\hline & & Chafe & 2 & 0 & 0 & 51 & 1 & 0.056 \\
\hline & & Burst & 5 & 0 & 0 & 0 & 5 & 0.500 \\
\hline & \multirow[t]{5}{*}{ VE } & Swim & 6995 & 2 & 0 & 3 & 0 & 0.001 \\
\hline & & HS & 4 & 17 & 0 & 1 & 1 & 0.261 \\
\hline & & Rest & 4 & 0 & 84 & 0 & 0 & 0.045 \\
\hline & & Chafe & 2 & 0 & 0 & 51 & 1 & 0.056 \\
\hline & & Burst & 2 & 1 & 0 & 0 & 7 & 0.300 \\
\hline
\end{tabular}

Rows indicate actual observations and columns represent predicted behaviours

Values in italic are correctly classified behavioural observations

$L R$ logistic regression, $A N N$ artificial neural network, $R F G$ random forest Gini, $R F E$ random forest entropy, $G B$ gradient tree boosting, $V E$ voting ensemble, $H S$ headshakes

\section{Discussion}

The main aim of this study was to develop ML models for converting ADL-derived features into behaviours, and to discern which ML classifiers performed best, for prediction of behaviours in the wild. Using the headshake class, we investigated whether the predictions, when considered in the context of relevant abiotic variables, would be suitable for drawing biologically relevant conclusions.

\section{Voting ensemble classifier}

It is important to consider the purpose of the classifier when establishing rare behaviours (i.e., feeding or burst events). If the goal is, as in this case, to investigate patterns of activity, an increased number of false positive predictions assigned to a rare class can obscure patterns in behaviour. For this reason, although the recall of the RF models was better than the other base learners and VE, the lack of precision made this model impractical as a standalone classifier, e.g., the RFE model predicted 209\% of the actual number of headshakes in the test set. Similarly, increased precision but poor recall, as in the case of the ANN classifier for the headshaking class, may result in a loss of 'true' information, and less clarity in behavioural patterns.

Class error output in all models was highest for the two rarest classes -0.26 and 0.30 for headshaking and burst 
Table 6 Performance metrics of base learner models and voting ensemble model

\begin{tabular}{|c|c|c|c|c|c|c|c|c|}
\hline Model & Class & $\mathrm{TP}$ & FP & FN & Precision & Recall & Class $F$-measure & $\begin{array}{l}\text { Macro- } \\
\text { averaged } \\
F \text {-measure }\end{array}$ \\
\hline \multirow[t]{5}{*}{ LR } & Swim & 6984 & 22 & 16 & 0.997 & 0.998 & 0.997 & 0.723 \\
\hline & HS & 16 & 17 & 7 & 0.485 & 0.696 & 0.571 & \\
\hline & Rest & 73 & 2 & 15 & 0.973 & 0.830 & 0.896 & \\
\hline & Chafe & 48 & 9 & 6 & 0.842 & 0.889 & 0.865 & \\
\hline & Burst & 2 & 2 & 8 & 0.500 & 0.200 & 0.286 & \\
\hline \multirow[t]{5}{*}{ ANN } & Swim & 6995 & 19 & 5 & 0.997 & 0.999 & 0.998 & 0.802 \\
\hline & HS & 11 & 7 & 12 & 0.611 & 0.478 & 0.537 & \\
\hline & Rest & 81 & 1 & 7 & 0.988 & 0.920 & 0.953 & \\
\hline & Chafe & 44 & 2 & 10 & 0.957 & 0.815 & 0.880 & \\
\hline & Burst & 8 & 7 & 2 & 0.533 & 0.800 & 0.640 & \\
\hline \multirow[t]{5}{*}{ RFG } & Swim & 6969 & 6 & 31 & 0.999 & 0.996 & 0.997 & 0.810 \\
\hline & HS & 19 & 25 & 4 & 0.432 & 0.826 & 0.567 & \\
\hline & Rest & 85 & 0 & 3 & 1.000 & 0.966 & 0.983 & \\
\hline & Chafe & 49 & 10 & 5 & 0.831 & 0.907 & 0.867 & \\
\hline & Burst & 7 & 5 & 3 & 0.583 & 0.700 & 0.636 & \\
\hline \multirow[t]{5}{*}{ RFE } & Swim & 6968 & 6 & 32 & 0.999 & 0.995 & 0.997 & 0.804 \\
\hline & HS & 18 & 30 & 5 & 0.375 & 0.783 & 0.507 & \\
\hline & Rest & 85 & 0 & 3 & 1.000 & 0.966 & 0.983 & \\
\hline & Chafe & 48 & 9 & 6 & 0.842 & 0.889 & 0.865 & \\
\hline & Burst & 7 & 4 & 3 & 0.636 & 0.700 & 0.667 & \\
\hline \multirow[t]{5}{*}{ GB } & Swim & 6995 & 16 & 5 & 0.998 & 0.999 & 0.999 & 0.856 \\
\hline & HS & 16 & 2 & 7 & 0.889 & 0.696 & 0.780 & \\
\hline & Rest & 84 & 0 & 4 & 1.000 & 0.955 & 0.977 & \\
\hline & Chafe & 51 & 4 & 3 & 0.927 & 0.944 & 0.936 & \\
\hline & Burst & 5 & 2 & 5 & 0.714 & 0.500 & 0.588 & \\
\hline \multirow[t]{5}{*}{ VE } & Swim & 6995 & 12 & 5 & 0.998 & 0.999 & 0.999 & 0.888 \\
\hline & HS & 17 & 3 & 6 & 0.850 & 0.739 & 0.791 & \\
\hline & Rest & 84 & 0 & 4 & 1.000 & 0.955 & 0.977 & \\
\hline & Chafe & 51 & 4 & 3 & 0.927 & 0.944 & 0.936 & \\
\hline & Burst & 7 & 2 & 3 & 0.778 & 0.700 & 0.737 & \\
\hline
\end{tabular}

The values in italic show optimum values for each metric

$L R$ logistic regression, $A N N$ artificial neural network, $R F G$ random forest Gini, $R F E$ random forest entropy, $G B$ gradient tree boosting, $V E$ voting ensemble, $H S$ headshake class; $T P$ true positive, $F P$ false positive, $F N$ false negative
Table 7 Log-likelihoods scores for models investigating the occurrence of headshakes in lemon sharks in Bimini, Bahamas

\begin{tabular}{lc}
\hline Covariates & Log-likelihood \\
\hline $\mathrm{s}($ Hour $)+$ factor(Tide) + (Season) & $-1265.815^{\mathrm{a}}$ \\
$\mathrm{s}($ Hour $)+$ factor(Season) & -1268.462 \\
$\mathrm{~s}($ Hour $)+$ factor(Tide) & -1269.209 \\
$\mathrm{~s}($ Hour $)$ & -1271.833 \\
factor(Tide) + (Season) & -1300.566 \\
factor(Tide) & -1304.014 \\
factor(Season) & -1311.745 \\
\hline
\end{tabular}

${ }^{\mathrm{a}}$ Optimal model behaviours, respectively in the VE. This is largely due to the comparatively small class sizes, resulting in the misclassification of one event having an overall greater impact on error output. This is a reflection of the extensive time (and subsequent ADL battery, memory capacity and cost) required to obtain data on infrequent behaviours in the lemon shark prohibiting a larger sample size. Such difficulties will vary with model species. Additionally, no headshaking occurred during one of the eight feeding events recorded during captive trials, representing a false negative rate of $12.5 \%$. In the future, obtaining further records of feeding specifically would indicate the accuracy of the current false negative rate associated with this class (which may be related to prey size), whilst generally increasing records of rare behaviours 
Table 8 Results of the final binomial generalized additive model investigating the presence of headshaking by lemon sharks in Bimini, Bahamas

\begin{tabular}{|c|c|c|c|c|c|c|}
\hline Covariate & & edf & ref.df & $X^{2}$ & $p$ value & $R^{2}$ (adj.) \\
\hline Hour & & 5.903 & 8 & 73.82 & $\leq 0.05$ & \\
\hline- & Level & Coefficient & $\mathrm{SE}$ & $z$ value & - & \\
\hline \multirow[t]{4}{*}{ Tide } & Intercept & 0.576 & 0.212 & 2.722 & $\leq 0.05$ & 0.0829 \\
\hline & Flood & 0.012 & 0.132 & 0.090 & 0.929 & \\
\hline & High & -0.600 & 0.149 & -4.035 & $\leq 0.05$ & \\
\hline & Low & 0.028 & 0.144 & 0.197 & 0.844 & \\
\hline Season & Dry & -0.868 & 0.282 & -3.082 & $\leq 0.05$ & \\
\hline
\end{tabular}

Outcomes of the smoother hour include: covariate, effective degrees of freedom (edf), reference degrees of freedom (ref.df), Chi squared value $\left(\chi^{2}\right), p$ value. Outcomes of factors include covariate, level, coefficient, standard error (SE), $z$ value and $p$ value. The overall adjusted $R^{2}$ value is also displayed

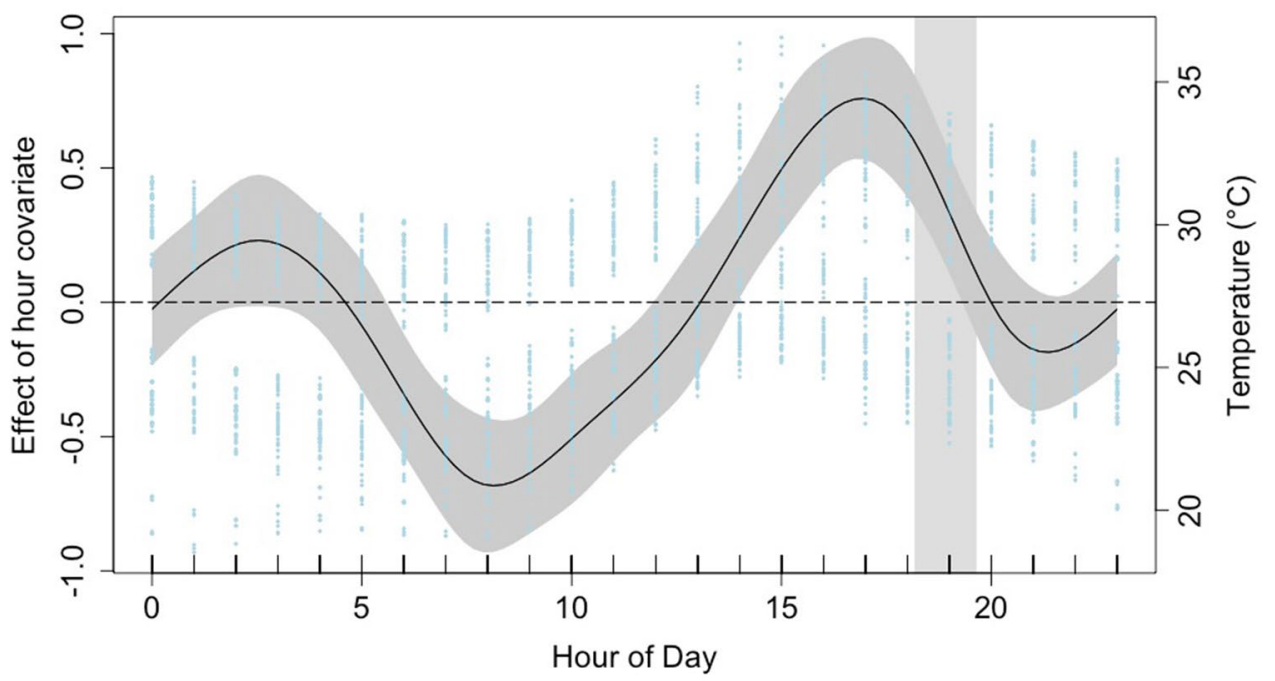

Fig. 3 Estimated smoother for the effect of hour of day on the probability of headshaking behaviour occurring by the juvenile lemon shark in Bimini, Bahamas. The lowest and highest probabilities of a headshake occurring are around 0800 and $1700 \mathrm{~h}$, respectively. Estimates are based on final binomial generalized additive mixed model. The solid line is the smoother. Dark grey shaded area surrounding the

would help overcome the class error output problem and likely improve classification performance by providing more events to train the model. The metrics used to assess ML performance should be considered in instances where correct classification of rare events is of interest. Accuracy is an often-referenced measure but can be misleading in such situations (Valverde-Albacete and Peláez-Moreno 2014).

Both the RF and GB models allow insight into the relative importance of predictor variables (Fig. S1). Overall, the models differed in their choices of important predictors, but agree that mean ODBA plays an important role. The difference in importance may be related to how the models spread the significance of correlated predictors, with GB models concentrating importance in a single variable and smoother represent $95 \%$ confidence intervals. The light grey shaded area represents the range of sunset times throughout the deployments. The dashed line represents the mean likelihood of a headshaking occurring. The blue dots represent mean hourly temperature $\left({ }^{\circ} \mathrm{C}\right)$, calculated from the temperature sensor in the acceleration data logger (ADL) packages, across all deployments

RF dispersing the importance across correlated variables (Freeman et al. 2015). ODBA is likely to be crucial in determining resting behaviour, where dynamic body movement ceases. Chafe, burst and prey capture behaviour exhibit increased ODBA values over steady swimming (Fig. 1).

Collecting ground-truthed data in realistic environmental conditions is important. In addition to being more likely to elicit natural behaviours, semi-enclosed pens are subject to ambient abiotic conditions and water movements that can inflate ODBA values obtained during rest periods (Whitney et al. 2010; Lear et al. 2017). Failure to account for these water movements during model training may result in misclassification of data obtained in the wild. Additionally, although not captured during captive 
trials and therefore not included as a behavioural class in this study, there is the potential for brief moments of gliding during swimming behaviour, which may be classified as resting. Therefore, resting predictions that occur sporadically and are of short duration should be considered with caution. For sharks that glide as part of their activity budget, the addition of vertical velocity as a feature vector may be beneficial for differentiating between resting and gliding. Considering headshakes in conjunction with burst-swimming events may also aid in distinguishing false positive headshakes, as these are likely to occur together as part of foraging behaviour. Burst-swimming events that are not succeeded by headshaking may represent a failed predation attempt or predator avoidance behaviour.

We have demonstrated that classification performance is dependent on the ML method applied, but it can also be affected by the number of classification categories and epoch length (Ladds et al. 2017). Although in this instance, the VE classifier performed better than the constituent base learners, there are some notes of caution for researchers looking to employ this method. First, the base learners employed here are not exhaustive and therefore the ability of this VE classifier to outperform other untested ML methods cannot be indicated. Second, the performance of the VE classifier has not yet been examined outside of our model species or across ontogeny and therefore we cannot attest to its ability to generalize beyond the conditions under which it was developed. Due to the vast range of body movement across the animal kingdom, it is unlikely a single method will provide optimum performance across all species (Ladds et al. 2017). Finally, the ML algorithms employed here do not account for auto-correlation which is expected in chronological acceleration data. Leos-Barajas et al. (2017) advise that whilst this may not matter in instances where the end goal is solely behavioural classification, using the output of such ML classifiers in subsequent statistical steps may render fraudulent results. The results of our study reflect ongoing observations around the Bimini Islands and as such, not accounting for serial-dependence in the classifier development stage does not appear to have impacted the results of our classifier application. However, this may not always be the case and inclusion of an autocorrelation feature vector may be required (e.g., Nathan et al. 2012; Ladds et al. 2017) or alternative models, such as hidden Markov models, which account for temporal dependency could be more applicable (Leos-Barajas et al. 2017; Dhir et al. 2017).

\section{Classifier application}

In this study, we selected headshaking behaviour to investigate whether the behavioural predictions made on wild data yielded biologically relevant results in relation to abiotic variables. During observations of wild and captive sharks, headshaking behaviour for juvenile lemon sharks at Bimini has only been witnessed as part of prey capture and therefore it is considered here as a proxy for successful foraging. Cortés and Gruber (1990) conducted an extensive stomach eversion study on lemon sharks from Bimini and Florida, USA. Using estimated time of consumption, they deemed that feeding in juvenile lemon sharks [ $43-83.7 \mathrm{~cm}$ precaudal length (PCL)] was asynchronous in relation to time of day and tide, and that they were opportunistic feeders. Here, we show that significantly fewer successful predations occurred during the high tide. This supports anecdotal evidence from Bimini, Bahamas, suggesting that feeding occurs more often over the low tides than the high tides (Guttridge 2009; Guttridge et al. 2012). Guttridge (2009) identified that many individuals sought refuge in a mangrove inlet from larger predators able to access the lagoon during high tides, e.g., large lemon sharks (Guttridge et al. 2012) and tiger sharks (Galeocerdo cuvier; Hansell et al. 2017). This tidally driven habitat selection is thought to be determined by anti-predator behaviour rather than increased foraging prospects, as only one hunting event was witnessed in the mangrove inlet refuge in more than 70 days of direct observations (Guttridge et al. 2012)

Conversely, Guttridge (2009) documented juvenile lemon sharks moving to more exposed areas during low tides, such as the lagoon, where four predations and 12 foragingrelated events (e.g., chasing fish) were witnessed during only 23 days of direct observations. Additionally, prey preference studies conducted at Bimini indicate juvenile lemon sharks feed preferentially both in terms of prey species and prey size, but can feed opportunistically when necessary (Newman et al. 2010, 2011). These contrasting findings indicate that the location of a nursery ground-even within a population-may affect feeding habits of juvenile lemon sharks, warranting further study.

Although studies conducted around the Bimini Islands versus those conducted in a laboratory conflict as to whether the juvenile lemon shark is predominantly nocturnal or crepuscular, rates of movement and metabolic rates are lowest during daylight hours (Morrissey and Gruber 1993; Nixon and Gruber 1988; Sundström et al. 2001). This is reflected in our results, where the incidence of successful predations is lowest during the morning and middle of the day. We found time of day to be a significant covariate affecting successful predations. Most feeding events occurred during early evening, close to sunset; this may be due to decreasing light levels. Sharks possess a reflective layer (tapetum lucidum) 
in the choroid, behind the retina, which enhances vision in low light conditions (Gardiner et al. 2012). Due to this visual adaptation, Sundström et al. (2001) suggest juvenile lemon sharks might hunt more actively during crepuscular or nocturnal periods, experiencing more frequent success during twilight. Papastamatiou et al. (2015) also found blacktip reef sharks (Carcharhinus melanopterus) are more active during the early evening and suggest this may be linked to increased foraging effort when they have a visual advantage over their prey.

Successful predations in the early evening may also be linked to diel temperature fluctuations. The body temperature of a poikilothermic shark, such as the lemon shark, is driven by ambient water temperature. Warmest daily water temperatures are experienced in the North Sound and Bonefish Hole nurseries during mid-afternoon, at $~ 1500 \mathrm{~h}$ (DiGirolamo et al. 2012; Fig. 3), approximately two hours before the highest presence of successful predations occur (Fig. 3). Blacktip reef sharks were most active as body temperatures began cooling after reaching their warmest temperatures for the day (Papastamatiou et al. 2015). The authors hypothesised that as predator escape responses scale at a greater rate with temperature than attack rates, blacktip reef sharks may exploit the higher thermal inertia that their body size confers, keeping their body temperature elevated for longer than their prey, increasing chances of successful predation. This may also apply to the juvenile lemon shark.

Significantly fewer incidents of predations occur during the dry season than in the wet season (Table 8), which falls in line with other findings (e.g., juvenile lemon sharks grow faster during the wet season; Gruber unpublished data). Clark (1959) found lemon sharks in semi-captive pens consumed less throughout the colder months, when temperatures fell below $24{ }^{\circ} \mathrm{C}$. During our deployments, ADL temperature loggers recorded a range of $26.3-36.6^{\circ} \mathrm{C}$ $(\bar{x}=30.47)$ and $18.5-29.2{ }^{\circ} \mathrm{C}(\bar{x}=24.10)$ during the wet and dry season, respectively. It is, therefore, expected that metabolic demands would be higher during the wet season (Lear et al. 2017) and energy intake would need to be augmented to meet these demands, whilst still allowing energy for somatic growth. This may be the result of increased foraging effort and/or greater prey abundance.

A further consideration is the attachment site of ADLs for the behaviour in question. In this case, we were interested in overall behaviour exhibited by juvenile lemon sharks, not only feeding events. Placement of mandible ADLs have been used to successfully identify foraging in marine mammals (e.g., Weddell seals [(Leptonychotes weddellii), Naito et al. 2010] and Stellar sea lions [(Eumetopias jubatus), Viviant et al. 2010], loggerhead turtles [(Caretta caretta), Okuyama et al. 2010] and the common carp [(Cyprinus carpio), Makiguchi et al. 2012]). Although the suction-feeding mechanisms differ between ray-finned fishes and elasmobranch fishes (Wilga et al. 2007), the latter study is of particular interest in relation to elasmobranchs that employ suction feeding as their principal feeding mode (e.g., the nurse shark (Ginglymostoma cirratum) and whitespotted bamboo sharks (Chiloscyllium plagiosum), which may not be readily distinguished through a dorsally mounted accelerometer.

In this instance, the behavioural classifications were binned as presence/absence by hour, as this was sufficient to demonstrate the application of the classifier to draw ecologically relevant conclusions. It illuminated patterns in the predatory behaviour of juvenile lemon sharks in relation to diel- and tidal cycles, as well as season. ADLs are capable though of providing fine-scale information and the timewindow should allow appropriate resolution for the question being addressed. One potential drawback to modelling the presence or absence of headshaking in hourly bins is that multiple prey captures within an hour and a single prey capture event would contribute to the analysis equally. However, this method was selected due to the variation in time spent prey-handling a single item. It may be beneficial for future studies to use mandible accelerometers to address whether there is a correlation between prey size and headshaking duration or intensity and whether prey handling techniques vary with prey type to allow for more quantitative analysis. Accelerometers have been used to identify feeding according to prey type in the white-streaked grouper [(Epinephelus ongus); Kawabata et al. 2014] and red-spotted grouper [(Epinephelus akaara); Horie et al. 2017] differentiating between shrimp, fish and crab. Although teleosts form most of the juvenile lemon shark diet, they have also been documented with crustaceans, other elasmobranch species [whiptail stingrays (Dasyatidae)], molluscs and annelidas in their stomachs (Newman et al. 2010).

Identification of behaviours exhibited in the wild allows construction of activity budgets. Accelerometer derived ODBA values have proven to be a valuable proxy for energy expenditure for many species, including teleosts (Wright et al. 2014; Metcalfe et al. 2016) and elasmobranchs (Gleiss et al. 2010; Lear et al. 2017). Once this relationship is established, pairing behavioural states with concurrent ODBA values can provide activity specific metabolic rates for deriving time-energy budgets for animals in situ. This was unattainable for aquatic species prior to the development of ADLs and now allows insight into the energetic costs of behavioural decisions, which have implications for fitness. Therefore, the mean daily field metabolic rate will be sensitive to changes in the activity budget (Jodice et al. 2003), which may shift because of human disturbance (e.g., wildlife watching, Constantine et al. 2004; Christiansen et al. 2013; Barnett et al. 2016), natural disturbance (e.g., climate events), seasons (Hanya 2004), habitat quality and food availability (Wauters et al. 1992; Li and Rogers 2004). In future studies, quantitative values of field metabolism (Lear 
et al. 2017) and relative feeding rates may allow for broad intra-species comparisons across climatic zones and environments with varying anthropogenic disturbance.

In conclusion, this study demonstrates the utility of a voting ensemble ML algorithm and its effectiveness as a classifier for predicting behaviours from accelerometer data. ML techniques are, and will continue to be, increasingly relied upon as accelerometer technology develops and the high information content they can obtain grows. This study indicates why selection of the most appropriate ML algorithm requires careful consideration of classifier application to allow for meaningful subsequent modelling. The precision and recall value for each class predicted by the VE model was not necessarily greater than the base-learners. However, the overall performance was superior by obtaining a balance of good recall, and model precision. This careful classifier development allowed for modelling of a behaviour against abiotic factors, showing that time of day, tidal phase and season are all significant factors in predicting feeding by the lemon shark. In doing so, it has provided empirical evidence that explains observations from numerous studies and has presented insight into the feeding ecology of the juvenile lemon shark.

Acknowledgements Bimini Biological Field Station Foundation (BBFSF) thanks the Department of Fisheries of the Commonwealth of the Bahamas for granting a Permit to Conduct Scientific Research (MAF/LIA/22). BBFSF also thanks their staff, volunteers and generous sponsors: Guy Harvey Ocean Foundation, Save our Seas Foundation, Composite Research boats, Mercury Engines and Davey Marine who enable ongoing research at the station. Any opinion, findings, and conclusions or recommendations expressed in this material are those of the authors and do not necessarily reflect the views of the National Science Foundation.

Funding This study was funded by the National Science Foundation Grants 1156145 (to SHG) and 1156141 (to NMW and ACG) under which this study was funded as part of a larger project.

\section{Compliance with ethical standards}

Conflict of interest The authors declare that they have no conflict of interest.

Ethical approval All applicable international, national, and/or institutional guidelines for the care and use of animals were followed. All procedures performed in studies involving animals were in accordance with the ethical standards of the institution or practice at which the studies were conducted (University of Miami Institutional Animal Care and Use Committee (IACUC), Protocol Number 12-030).

Open Access This article is distributed under the terms of the Creative Commons Attribution 4.0 International License (http://creativeco mmons.org/licenses/by/4.0/), which permits unrestricted use, distribution, and reproduction in any medium, provided you give appropriate credit to the original author(s) and the source, provide a link to the Creative Commons license, and indicate if changes were made.

\section{References}

Allen AN, Goldbogen JA, Friedlaender AS, Calambokidis J (2016) Development of an automated method of detecting stereotyped feeding events in multisensor data from tagged rorqual whales. Ecol Evol 6:7522-7535. https://doi.org/10.1002/ece3.2386

Barley SC, Meekan MG, Meeuwig JJ (2017) Species diversity, abundance, biomass, size and trophic structure of fish on coral reefs in relation to shark abundance. Mar Ecol Prog Ser 565:163-179. https://doi.org/10.3354/meps11981

Barnett A, Payne NL, Semmens JM, Fitzpatrick R (2016) Ecotourism increases the field metabolic rate of whitetip reef sharks. Biol Conserv 199:132-136. https://doi.org/10.1016/j.bioco n.2016.05.009

Battaile BC, Sakamoto KQ, Nordstrom CA, Rosen DA, Trites AW (2015) Accelerometers identify new behaviors and show little difference in the activity budgets of lactating northern fur seals (Callorhinus ursinus) between breeding islands and foraging habitats in the Eastern Bering Sea. PLoS One 10:e0118761. https ://doi.org/10.1371/journal.pone.0118761

Bidder OR, Campbell HA, Gómez-Laich A, Urgé P, Walker J, Cai Y, Gao L, Quintana F, Wilson RP (2014) Love thy neighbour: automatic animal behavioural classification of acceleration data using the k-nearest neighbour algorithm. PLoS One 9:e88609. https://doi.org/10.1371/journal.pone.0088609

Bograd SJ, Block BA, Costa DP, Godley BJ (2010) Biologging technologies: new tools for conservation. introduction. Endanger Species Res 10:1-7. https://doi.org/10.3354/esr00269

Bouyoucos IA, Montgomery DW, Brownscombe JW, Cooke SJ, Suski CD, Mandelman JW, Brooks EJ (2017) Swimming speeds and metabolic rates of semi-captive juvenile lemon sharks (Negaprion brevirostris, Poey) estimated with acceleration biologgers. $\mathrm{J}$ Exp Mar Biol Ecol 486:245-254. https://doi.org/10.1016/j.jembe .2016 .10 .019

Breiman L, Friedman J, Stone CJ, Olshen RA (1984) Classification and regression trees. CRC Press, Wadsworth

Brown DD, Kays R, Wikelski M, Wilson R, Klimley AP (2013) Observing the unwatchable through acceleration logging of animal behavior. Anim Biotelem 1:20. https://doi. org/10.1186/2050-3385-1-20

Bush A (2003) Diet and diel feeding periodicity of juvenile scalloped hammerhead sharks, Sphyrna lewini, in Kāne'ohe Bay, Ō'ahu, Hawai'i. Environ Biol Fish 67:1-11. https://doi. org/10.1023/A:102443870

Campbell HA, Gao L, Bidder OR, Hunter J, Franklin CE (2013) Creating a behavioural classification module for acceleration data: using a captive surrogate for difficult to observe species. J Exp Biol 216:4501-4506. https://doi.org/10.1242/jeb.089805

Carroll G, Slip D, Jonsen I, Harcourt R (2014) Supervised accelerometry analysis can identify prey capture by penguins at sea. J Exp Biol 217:4295-4302. https://doi.org/10.1242/jeb.113076

Casale P, Pujol O, Radeva P (2011) Human Activity Recognition from Accelerometer Data Using a Wearable Device. In: Vitrià J, Sanches JM, Hernández M (eds) Pattern Recognition and Image Analysis. IbPRIA 2011. Lecture Notes in Computer Science, vol 6669. Springer, Berlin, Heidelberg, pp 289-296. https://doi. org/10.1007/978-3-642-21257-4_36

Catal C, Tufekci S, Pirmit E, Kocabag G (2015) On the use of ensemble of classifiers for accelerometer-based activity recognition. Appl Soft Comput 37:1018-1022. https://doi.org/10.1016/j. asoc.2015.01.025

Chapman DD, Babcock EA, Gruber SH, Dibattista JD, Franks BR, Kessel SA, Guttridge T, Pikitch EK, Feldheim KA (2009) Longterm natal site-fidelity by immature lemon sharks (Negaprion 
brevirostris) at a subtropical island. Mol Ecol 18:3500-3507. https://doi.org/10.1111/j.1365-294X.2009.04289.x

Chen C, Liaw A, Breiman L (2004) Using random forest to learn imbalanced data. Tech. Rep. 666, Statistics Department, University of California, Berkeley

Chessa S, Micheli A, Pucci R, Hunter J, Carroll G, Harcourt R (2017) A comparative analysis of SVM and IDNN for identifying penguin activities. Appl Artif Intell. https://doi.org/10.1080/08839 514.2017.1378162

Chimienti M, Cornulier T, Owen E, Bolton M, Davies IM, Travis JM, Scott BE (2016) The use of an unsupervised learning approach for characterizing latent behaviors in accelerometer data. Ecol Evol 6:727-741. https://doi.org/10.1002/ece3.1914

Christiansen F, Rasmussen MH, Lusseau D (2013) Inferring activity budgets in wild animals to estimate the consequences of disturbances. Behav Ecol 24:1415-1425. https://doi.org/10.1093/ beheco/art086

Clark E (1959) Instrumental conditioning of lemon sharks. Science 130:217-218

Constantine R, Brunton DH, Dennis T (2004) Dolphin-watching tour boats change bottlenose dolphin (Tursiops truncatus) behaviour. Biol Conserv 117:299-307. https://doi.org/10.1016/j.bioco n.2003.12.009

Cooke SJ (2008) Biotelemetry and biologging in endangered species research and animal conservation: relevance to regional, national, and IUCN red list threat assessments. Endanger Species Res 4:165-185. https://doi.org/10.3354/esr00063

Cooke SJ, Hinch SG, Wikelski M, Andrews RD, Kuchel LJ, Wolcott TG, Butler PJ (2004) Biotelemetry: a mechanistic approach to ecology. Trends Ecol Evol 19:334-343. https://doi.org/10.1016/j. tree.2004.04.003

Cortés E (1999) Standardized diet compositions and trophic levels of sharks. ICES J Mar Sci 56:707-717. https://doi.org/10.1006/ jmsc. 1999.0489

Cortés E, Gruber SH (1990) Diet, feeding habits and estimates of daily ration of young lemon sharks, Negaprion brevirostris (Poey). Copeia. https://doi.org/10.2307/1445836

Cortés E, Gruber SH (1994) Effect of ration size on growth and gross conversion efficiency of young lemon sharks, Negaprion brevirostris. J Fish Biol 44:331-341. https://doi. org/10.1111/j.1095-8649.1994.tb01210.x

Dhir N, Wood F, Vákár M, Markham A, Wijers M, Trethowan P, Du Preez B, Loveridge A, MacDonald D (2017) Interpreting lion behaviour with nonparametric probabilistic programs

Digirolamo AL, Gruber SH, Pomory C, Bennett WA (2012) Diel temperature patterns of juvenile lemon sharks Negaprion brevirostris, in a shallow-water nursery. J Fish Biol 80:1436-1448. https ://doi.org/10.1111/j.1095-8649.2012.03263.x

Diosdado JAV, Barker ZE, Hodges HR, Amory JR, Croft DP, Bell NJ, Codling EA (2015) Classification of behaviour in housed dairy cows using an accelerometer-based activity monitoring system. Anim Biotelem 3:15. https://doi.org/10.1186/s40317-015-0045-8

Dutta R, Smith D, Rawnsley R, Bishop-Hurley G, Hills J, Timms G, Henry D (2015) Dynamic cattle behavioural classification using supervised ensemble classifiers. Comput Electron Agric 111:1828. https://doi.org/10.1016/j.compag.2014.12.002

Estrada JA, Rice AN, Lutcavage ME, Skomal GB (2003) Predicting trophic position in sharks of the north-west atlantic ocean using stable isotope analysis. J Mar Biol Assoc UK 83:1347-1350. https://doi.org/10.1017/s0025315403008798

Forsman A (2015) Rethinking phenotypic plasticity and its consequences for individuals, populations and species. Heredity 115(4):276. https://doi.org/10.1038/hdy.2014.92

Freeman EA, Moisen GG, Coulston JW, Wilson BT (2015) Random forests and stochastic gradient boosting for predicting tree canopy cover: comparing tuning processes and model performance 1. Can J For Res 46:323-339. https://doi. org/10.1139/cjfr-2014-0562

Friedman JH (2001) Greedy function approximation: a gradient boosting machine. Ann Statist 29:1189-1232. https://doi:10.1214/ aos/1013203451

Gardiner JM, Heuter RE, Maruska KP, Sisneros JA, Casper BM, Mann DA, Demski LS (2012) Sensory physiology and behavior of elasmobranchs. In: Carrier JC, Musick JA, Heithaus MR (eds) Biology of sharks and their relatives, 2nd edn. CRC Press, Florida, pp 349-402

Gleiss AC, Gruber SH, Wilson RP (2009a) Multi-channel data-logging: towards determination of behaviour and metabolic rate in freeswimming sharks. In: Nielsen JL, Arrizabalaga H, Fragoso N, Hobday A, Lutcavage M, Sibert J (eds) Tagging and tracking of marine animals with electronic devices. Springer, New York

Gleiss AC, Norman B, Liebsch N, Francis C, Wilson RP (2009b) A new prospect for tagging large free-swimming sharks with motion-sensitive data-loggers. Fish Res 97:11-16. https://doi. org/10.1016/j.fishres.2008.12.012

Gleiss AC, Dale JJ, Holland KN, Wilson RP (2010) Accelerating estimates of activity-specific metabolic rate in fishes: testing the applicability of acceleration data-loggers. J Exp Mar Biol Ecol 385:85-91. https://doi.org/10.1016/j.jembe.2010.01.012

Gleiss AC, Norman B, Wilson RP (2011a) Moved by that sinking feeling: variable diving geometry underlies movement strategies in whale sharks. Funct Ecol 25:595-607. https://doi.org/10.111 1/j.1365-2435.2010.01801.x

Gleiss AC, Wilson RP, Shepard ELC (2011b) Making overall dynamic body acceleration work: on the theory of acceleration as a proxy for energy expenditure. Methods Ecol Evol 2:23-33. https://doi. org/10.1111/j.2041-210X.2010.00057.x

Gleiss AC, Wright S, Liebsch N, Wilson RP, Norman B (2013) Contrasting diel patterns in vertical movement and locomotor activity of whale sharks at Ningaloo reef. Mar Biol 160:2981-2992. https ://doi.org/10.1007/s00227-013-2288-3

Gleiss AC, Morgan DL, Whitty JM, Keleher JJ, Fossette S, Hays GC (2017) Are vertical migrations driven by circadian behaviour? Decoupling of activity and depth use in a large riverine elasmobranch, the freshwater sawfish (Pristis pristis). Hydrobiologia 787:181-191. https://doi.org/10.1007/s10750-016-2957-6

Graf PM, Wilson RP, Qasem L, Hackländer K, Rosell F (2015) The use of acceleration to code for animal behaviours; a case study in free-ranging eurasian beavers castor fiber. PLoS One 10:e0136751. https://doi.org/10.1371/journal.pone.0136751

Grier JW (1984) Biology of animal behavior. Times Mirror/Mosby College Publishing, St. Missouri

Gruber SH (1982) Role of the lemon shark, Negaprion brevirostris (Poey) as a predator in the tropical marine environment: a multidisciplinary study. Flo Scient 45:46-75

Gruber SH, De Marignac JR, Hoenig JM (2001) Survival of juvenile lemon sharks at Bimini, Bahamas, estimated by mark-depletion experiments. Trans Am Fish Soc 130:376-384. https://doi. org/10.1577/1548-8659(2001)130<0376:sojlsa >2.0.co;2

Grünewälder S, Broekhuis F, Macdonald DW, Wilson AM, McNutt JW, Shawe-Taylor J, Hailes S (2012) Movement activity based classification of animal behaviour with an application to data from cheetah (Acinonyx jubatus). PLoS One 7:e49120. https:// doi.org/10.1371/journal.pone.0049120

Guttridge TL (2009) The social organisation and behaviour of the juvenile lemon shark, Negaprion brevirostris. Doctoral thesis, University of Leeds, UK

Guttridge TL, Gruber SH, Gledhill KS, Croft DP, Sims DW, Krause J (2009) Social preferences of juvenile lemon sharks, Negaprion brevirostris. Anim Behav 78(2):543-548. https://doi. org/10.1016/j.anbehav.2009.06.009 
Guttridge T, Gruber S, Franks B, Kessel S, Gledhill K, Uphill J, Krause J, Sims D (2012) Deep danger: intra-specific predation risk influences habitat use and aggregation formation of juvenile lemon sharks Negaprion brevirostris. Mar Ecol Prog Ser 445:279-291. https://doi.org/10.3354/meps09423

Hansell AC, Kessel ST, Brewster LR, Cadrin SX, Gruber SH, Skomal GB, Guttridge TL (2017) Local indicators of abundance and demographics for the coastal shark assemblage of the eastern waters of Bimini, Bahamas. Fish Res. https://doi.org/10.1016/j. fishres.2017.09.016

Hanya G (2004) Seasonal variations in the activity budget of japanese macaques in the coniferous forest of yakushima: effects of food and temperature. Am J Primatol 63:165-177. https:// doi.org/10.1002/ajp.20049

Hastie TJ, Tibshirani R (1990) Generalized additive models. Encycl Stat Sci. https://doi.org/10.1002/0471667196.ess0297.pub2

Hastie TJ, Tibshirani R, Friedman J (2009) The Elements of Statistical Learning: data Mining, Inference, and Prediction. 2nd edn. Springer, New York

Heithaus MR, Frid A, Wirsing AJ, Worm B (2008) Predicting ecological consequences of marine top predator declines. Trends Ecol Evol 23:202-210. https://doi.org/10.1016/j. tree.2008.01.003

Horie J, Mitamura H, Ina Y, Mashino Y, Noda T, Moriya K, Arai N, Sasakura T (2017) Development of a method for classifying and transmitting high-resolution feeding behavior of fish using an acceleration pinger. Anim Biotelem 5:12. https://doi. org/10.1186/s40317-017-0127-x

Jodice P, Roby D, Suryan R, Irons D, Kaufman A, Turco K, Visser G (2003) Variation in energy expenditure among blacklegged kittiwakes: effects of activity-specific metabolic rates and activity budgets. Physiol Biochem Zool 76:375-388. https ://doi.org/10.1086/375431

Johnson JB, Omland KS (2004) Model selection in ecology and evolution. Trends Ecol Evol 19:101-108. https://doi.org/10.1016/j. tree.2003.10.013

Kawabata Y, Noda T, Nakashima Y, Nanami A, Sato T, Takebe T, Mitamura H, Arai N, Yamaguchi T, Soyano K (2014) Use of a gyroscope/accelerometer data logger to identify alternative feeding behaviours in fish. J Exp Biol 217:3204-3208. https:// doi.org/10.1242/jeb.108001

Kiani K, Snijders CJ, Gelsema ES (1998) Recognition of daily life motor activity classes using an artificial neural network. Arch Phys Med Rehabil 79:147-154. https://doi.org/10.1016/S0003 -9993(98)90291-X

Klimley AP, Anderson SD, Pyle P, Henderson RP (1992) Spatiotemporal patterns of white shark (Carcharodon carcharias) predation at the South Farallon Islands, California. Copeia. https:// doi.org/10.2307/1446143

Ladds MA, Thompson AP, Kadar JP, Slip D, Hocking D, Harcourt R (2017) Super machine learning: improving accuracy and reducing variance of behaviour classification from accelerometry. Anim Biotelem 5:8. https://doi.org/10.1186/s4031 7-017-0123-1

Lear KO, Whitney NM, Brewster LR, Morris JJ, Hueter RE, Gleiss AC (2017) Correlations of metabolic rate and body acceleration in three species of coastal sharks under contrasting temperature regimes. J Exp Biol. https://doi.org/10.1242/jeb.14699 3

Leos-Barajas V, Photopoulou T, Langrock R, Patterson TA, Watanabe YY, Murgatroyd M, Papastamatiou YP (2017) Analysis of animal accelerometer data using hidden markov models. Methods Ecol Evol 8:161-173. https://doi. org/10.1111/2041-210X.12657
Li Z, Rogers E (2004) Habitat quality and activity budgets of whiteheaded langurs in fusui, China. Int J Primatol 25:41-54. https ://doi.org/10.1023/B:IJOP.0000014644.36333.94

Lima SL, Dill LM (1990) Behavioral decisions made under the risk of predation: a review and prospectus. Can J Zool 68:619-640. https://doi.org/10.1139/z90-092

Luštrek M, Kaluža B (2009) Fall detection and activity recognition with machine learning. Informatica 33:205-212

Makiguchi Y, Sugie Y, Kojima T, Naito Y (2012) Detection of feeding behaviour in common carp cyprinus carpio by using an acceleration data logger to identify mandibular movement. J Fish Biol 80:2345-2356. https://doi.org/10.1111/j.1095-8649.2012.03293 .X

Martiskainen P, Järvinen M, Skön JP, Tiirikainen J, Kolehmainen M, Mononen J (2009) Cow behaviour pattern recognition using a three-dimensional accelerometer and support vector machines. Appl Anim Behav Sci 119:32-38. https://doi.org/10.1016/j.appla nim.2009.03.005

McClune DW, Marks NJ, Wilson RP, Houghton JD, Montgomery IW, McGowan NE, Gormley E, Scantlebury M (2014) Tri-axial accelerometers quantify behaviour in the Eurasian badger (Meles meles): towards an automated interpretation of field data. Anim Biotelem 2:5. https://doi.org/10.1186/2050-3385-2-5

McNamara JM, Houston AI (1996) State-dependent life histories. Nature 380:215-221

Metcalfe JD, Wright S, Tudorache C, Wilson RP (2016) Recent advances in telemetry for estimating the energy metabolism of wild fishes. J Fish Biol 88:284-297. https://doi.org/10.1111/ jfb. 12804

Morrissey JF, Gruber SH (1993) Home range of juvenile lemon sharks, Negaprion brevirostris. Copeia 1993:425-434. https:// doi.org/10.2307/1447141

Myrberg AA Jr, Gruber SH (1974) The behavior of the bonnethead shark, sphyrna tiburo. Copeia. https://doi.org/10.2307/1442530

Naito Y, Bornemann H, Takahashi A, McIntyre T, Plötz J (2010) Finescale feeding behavior of weddell seals revealed by a mandible accelerometer. Polar Sci 4:309-316. https://doi.org/10.1016/j. polar.2010.05.009

Nakamura I, Watanabe Y, Papastamatiou Y, Sato K, Meyer C (2011) Yo-yo vertical movements suggest a foraging strategy for tiger sharks galeocerdo cuvier. Mar Ecol Prog Ser 424:237-246. https ://doi.org/10.3354/meps08980

Nathan R, Spiegel O, Fortmann-Roe S, Harel R, Wikelski M, Getz WM (2012) Using tri-axial acceleration data to identify behavioral modes of free-ranging animals: general concepts and tools illustrated for griffon vultures. J Exp Biol 215:986-996. https:// doi.org/10.1242/jeb.058602

Newman S, Handy R, Gruber SH (2010) Diet and prey preference of juvenile lemon sharks Negaprion brevirostris. Mar Ecol Prog Ser 398:221-234. https://doi.org/10.3354/meps08334

Newman SP, Handy RD, Gruber SH (2011) Ontogenetic diet shifts and prey selection in nursery bound lemon sharks, Negaprion brevirostris, indicate a flexible foraging tactic. Environ Biol Fishes 95:115-126. https://doi.org/10.1007/s10641-011-9828-9

Nixon ASAJ, Gruber SH (1988) Diel metabolic and activity patterns of the lemon shark (Negaprion brevirostris). J Exp Zool 248:1-6. https://doi.org/10.1002/jez.1402480102

Okuyama J, Kawabata Y, Naito Y, Arai N, Kobayashi M (2010) Monitoring beak movements with an acceleration datalogger: a useful technique for assessing the feeding and breathing behaviors of sea turtles. Endanger Species Res 10:39-45. https://doi. org/10.3354/esr00215

Owen K, Dunlop RA, Monty JP, Chung D, Noad MJ, Donnelly D, Goldizen AW, Mackenzie T (2016) Detecting surface-feeding behavior by rorqual whales in accelerometer data. Mar Mammal Sci 32:327-348. https://doi.org/10.1111/mms.12271 
Özgür A, Özgür L, Güngör T (2005) Text categorization with classbased and corpus-based keyword selection. Proceeding 20th Internat. Symposium on computer and information sciences (ISCIS, 2005), Lecture notes in computer science, vol 3733. Springer, Berlin, pp 606-615

Papastamatiou YP, Watanabe YY, Bradley D, Dee LE, Weng K, Lowe CG, Caselle JE (2015) Drivers of daily routines in an ectothermic marine predator: hunt warm, rest warmer? PLoS One 10:e127807. https://doi.org/10.1371/journal.pone.0127807

Papastamatiou YP, Iosilevskii G, Leos-Barajas V, Brooks EJ, Howey LA, Chapman DD, Watanabe YY (2018) Optimal swimming strategies and behavioural plasticity of oceanic whitetip sharks. Sci Rep 8:551. https://doi.org/10.1038/s41598-017-18608-z

Payne NL, Iosilevskii G, Barnett A, Fischer C, Graham RT, Gleiss AC, Watanabe YY (2016) Great hammerhead sharks swim on their side to reduce transport costs. Nat Commun. https://doi. org/10.1038/ncomms12289

Pedregosa F, Varoquaux G, Gramfort A, Michel V, Thirion B, Grisel O, Blondel M, Prettenhofer P, Weiss R, Dubourg V (2011) Scikit-learn: machine learning in python. J Mach Learn Res 12:2825-2830

Rasher DB, Hoey AS, Hay ME (2017) Cascading predator effects in a Fijian coral reef ecosystem. Sci Rep UK 7:15684. https://doi. org/10.1038/s41598-017-15679-w

Resheff YS, Rotics S, Harel R, Spiegel O, Nathan R (2014) AcceleRater: a web application for supervised learning of behavioral modes from acceleration measurements. Mov Ecol 2:1. https:// doi.org/10.1186/s40462-014-0027-0

Rifkin R, Klautau A (2004) In defense of one-vs-all classification. J Mach Learn Res 5:101-141

Rutz C, Hays GC (2009) New frontiers in biologging science. Biol Lett 5:289-292. https://doi.org/10.1098/rsbl.2009.0089

Sakamoto KQ, Sato K, Ishizuka M, Watanuki Y, Takahashi A, Daunt F, Wanless S (2009) Can ethograms be automatically generated using body acceleration data from free-ranging birds? PLoS One 4:e5379. https://doi.org/10.1371/journal.pone.0005379

Schmidhuber J (2015) Deep learning in neural networks: an overview. Neural Netw. https://doi.org/10.1016/j.neunet.2014.09.003

Shepard E, Wilson R, Halsey L, Quintana F, Gómez Laich A, Gleiss A, Liebsch N, Myers A, Norman B (2008a) Derivation of body motion via appropriate smoothing of acceleration data. Aquat Biol 4:235-241. https://doi.org/10.3354/ab00104

Shepard E, Wilson R, Quintana F, Gómez Laich A, Liebsch N, Albareda D, Halsey L, Gleiss A, Morgan D, Myers A, Newman C, McDonald D (2008b) Identification of animal movement patterns using tri-axial accelerometry. Endanger Species Res 10:47-60. https://doi.org/10.3354/esr00084

Sokolova M, Lapalme G (2009) A systematic analysis of performance measures for classification tasks. Inf Process Manag 45:427-437. https://doi.org/10.1016/j.ipm.2009.03.002

Soltis J, Wilson RP, Douglas-Hamilton I, Vollrath F, King LE, Savage A (2012) Accelerometers in collars identify behavioral states in captive african elephants Loxodonta africana. Endanger Species Res 18:255-263. https://doi.org/10.3354/esr00452

Staudenmayer J, Pober D, Crouter S, Bassett D, Freedson P (2009) An artificial neural network to estimate physical activity energy expenditure and identify physical activity type from an accelerometer. J Appl Physiol 107:1300-1307. https://doi.org/10.1152/ japplphysiol.00465.2009

Stevens J, Bonfil R, Dulvy N, Walker P (2000) The effects of fishing on sharks, rays, and chimaeras (chondrichthyans), and the implications for marine ecosystems. ICES J Mar Sci 57:476-494. https ://doi.org/10.1006/jmsc.2000.0724

Sundström L, Gruber SH, Clermont SM, Correia J, de Marignac J, Morrissey JF, Lowrance CR, Thomassen L, Oliveira MT (2001) Review of elasmobranch behavioral studies using ultrasonic telemetry with special reference to the lemon shark, Negaprion brevirostris, around Bimini Islands, Bahamas. Environ Biol Fishes 60:225-250. https://doi. org/10.1007/978-94-017-3245-1_13

Sur M, Suffredini T, Wessells SM, Bloom PH, Lanzone M, Blackshire S, Sridhar S, Katzner T (2017) Improved supervised classification of accelerometry data to distinguish behaviors of soaring birds. PLoS One 12:e0174785. https://doi.org/10.1371/journ al.pone. 0174785

Tanha J, Van Someren M, de Bakker M, Bouteny W, ShamounBaranesy J, Afsarmanesh H (2012) Multiclass semi-supervised learning for animal behavior recognition from accelerometer data. IEEE International Conference on Tools with Artificial Intelligence, vol 1, pp 690-697. https://doi.org/10.1109/ictai .2012 .98

Valletta JJ, Torney C, Kings M, Thornton A, Madden J (2017) Applications of machine learning in animal behaviour studies. Anim Behav 124:203-220. https://doi.org/10.1016/j.anbeh av.2016.12.005

Valverde-Albacete FJ, Peláez-Moreno C (2014) 100\% classification accuracy considered harmful: the normalized information transfer factor explains the accuracy paradox. PLoS One 9:e84217. https://doi.org/10.1371/journal.pone.0084217

Viviant M, Trites AW, Rosen DA, Monestiez P, Guinet C (2010) Prey capture attempts can be detected in steller sea lions and other marine predators using accelerometers. Polar Biol 33:713-719. https://doi.org/10.1007/s00300-009-0750-y

Walker JS, Jones MW, Laramee RS, Bidder OR, Williams HJ, Scott R, Shepard ELC, Wilson RP (2015) TimeClassifier: a visual analytic system for the classification of multi-dimensional time series data. Vis Comput 31:1067-1078. https://doi.org/10.1007/ s00371-015-1112-0

Wang Y, Nickel B, Rutishauser M, Bryce CM, Williams TM, Elkaim G, Wilmers CC (2015) Movement, resting, and attack behaviors of wild pumas are revealed by tri-axial accelerometer measurements. Mov Ecol 3:1. https://doi.org/10.1186/s40462-015-0030-0

Wasserman L (2000) Bayesian model selection and model averaging. J Math Psychol 44:92-107. https://doi.org/10.1006/ jmps.1999.1278

Watanabe YY, Lydersen C, Fisk AT, Kovacs KM (2012) The slowest fish: swim speed and tail-beat frequency of greenland sharks. J Exp Mar Biol Ecol 426-427:5-11. https://doi.org/10.1016/j. jembe.2012.04.021

Wauters L, Swinnen C, Dhondt A (1992) Activity budget and foraging behaviour of red squirrels (Sciurus vulgaris) in coniferous and deciduous habitats. J Zool 227:71-86. https://doi. org/10.1111/j.1469-7998.1992.tb04345.x

Wetherbee BM, Gruber SH, Ramsey AL (1987) X-radiographic observations of food passage through digestive tracts of lemon sharks. Trans Am Fish Soc 116:763-767

Wetherbee BM, Gruber SH, Cortes E (1990) Diet, feeding habits, digestion, and consumption in sharks with special reference to the lemon shark, Negaprion brevirostris. In Pratt HL Jr, Harold L, Gruber SH, Toru T (eds) Elasmobranchs as living resources: advances in the biology, ecology, systematics, and the status of the fisheries. NOAA/National Marine Fisheries Service, (NOAA Technical Report NMFS, 90): 29

Whitney NM, Papastamatiou YP, Holland KN, Lowe CG (2007) Use of an acceleration data logger to measure diel activity patterns in captive whitetip reef sharks, triaenodon obesus. Aquat Living Resour 20:299-305. https://doi.org/10.1051/alr:2008006

Whitney NM, Pratt H, Pratt T, Carrier J (2010) Identifying shark mating behaviour using three-dimensional acceleration loggers. Endanger Species Res 10:71-82. https://doi.org/10.3354/esr00 247 
Whitney NM, White CF, Gleiss AC, Schwieterman GD, Anderson P, Hueter RE, Skomal GB (2016a) A novel method for determining post-release mortality, behavior, and recovery period using acceleration data loggers. Fish Res 183:210-221. https://doi. org/10.1016/j.fishres.2016.06.003

Whitney NM, Lear KO, Gaskins LC, Gleiss AC (2016b) The effects of temperature and swimming speed on the metabolic rate of the nurse shark (Ginglymostoma cirratum, bonaterre). J Exp Mar Biol Ecol 477:40-46. https://doi.org/10.1016/j.jembe .2015.12.009

Wilga CD, Motta PJ, Sanford CP (2007) Evolution and ecology of feeding in elasmobranchs. Integr Comp Biol 47:55-69. https:// doi.org/10.1093/icb/icm029

Williams H, Shepard E, Duriez O, Lambertucci S (2015) Can accelerometry be used to distinguish between flight types in soaring birds? Anim Biotelem 3:1. https://doi.org/10.1186/s4031 7-015-0077-0

Wilson RP, White CR, Quintana F, Halsey LG, Liebsch N, Martin GR, Butler PJ (2006) Moving towards acceleration for estimates of activity-specific metabolic rate in free-living animals: the case of the cormorant. J Anim Ecol 75:1081-1090. https://doi.org/10 $.1111 / \mathrm{j} .1365-2656.2006 .01127 . x$

Wright S, Metcalfe JD, Hetherington S, Wilson RP (2014) Estimating activity-specific energy expenditure in a teleost fish, using accelerometer loggers. Mar Ecol Prog Ser 496:19-32. https://doi. org/10.3354/meps 10528

Yoda K, Sato K, Niizuma Y, Kurita M, Bost C, Le Maho Y, Naito $Y$ (1999) Precise monitoring of porpoising behaviour of adelie penguins determined using acceleration data loggers. J Exp Biol 202:3121-3126

Zheng Y, Wong WK, Guan X, Trost S (2013) Physical activity recognition from accelerometer data using a multi-scale ensemble method. In: Proceedings of the 25th conference on innovative applications of artificial intelligence, Bellevue, Washington, DC, USA, 14-18 July

Zuur AFS, Ieno AA (2012) Zero inflated models and generalized linear mixed models with R. Highland Statistics Ltd 